\title{
3D-partition functions on the sphere: exact evaluation and mirror symmetry
}

\author{
Sergio Benvenuti ${ }^{1}$ Sara Pasquetti ${ }^{2}$ \\ ${ }^{1}$ Theoretical Physics Group, The Blackett Laboratory, \\ Imperial College, Prince Consort Road, London SW7 2AZ, UK \\ ${ }^{2}$ School of Physics, Queen Mary University of London, \\ Mile End Road, London E1 4NS, UK
}

\begin{abstract}
We study $\mathcal{N}=4$ quiver theories on the three-sphere. We compute partition functions using the localisation method by Kapustin et al. solving exactly the matrix integrals at finite $N$, as functions of mass and Fayet-Iliopoulos parameters. We find a simple explicit formula for the partition function of the quiver tail $T(S U(N))$. This formula opens the way for the analysis of star-shaped quivers and their mirrors (that are the Gaiotto-type theories arising from M5 branes on punctured Riemann surfaces). We provide non-perturbative checks of mirror symmetry for infinite classes of theories and find the partition functions of the $T_{N}$ theory, the building block of generalised quiver theories.
\end{abstract}




\section{Contents}

1 Introduction $\quad 1$

2 Our set-up $\quad 3$

2.1 The Kapustin-Willet-Yaakov matrix integrals 4

2.2 Abelian integrals: $U(1)$ with $N$ flavours 5

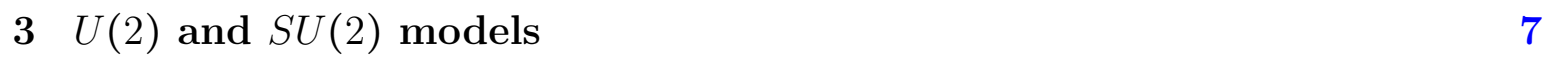

$3.1 S U(2)$ with K flavours $\quad 7$

3.2 The $D_{k}$ quiver 8

$3.3 S U(2) \times U(1)^{N}$ star shaped 11

4 Lagrangian theories $\quad 13$

$4.1 T(S U(N)) \quad 13$

4.1.1 Proof of the formula by induction $\quad 15$

4.2 Two maximal and one minimal puncture $\quad 16$

4.3 Two maximal and $k+2$ minimal 18

$\begin{array}{lll}5 & T_{N} \text { theories } & 21\end{array}$

5.1 The $T_{N}$ block 22

5.2 Consistency checks from S-duality invariance 24

6 Conclusions 27

\section{Introduction}

Over the last two years the new class of $4 d \mathcal{N}=2$ super-conformal gauge theories introduced by Gaiotto [1] has attracted much interest. This class of theories can be viewed as arising from a super-conformal $6 d$ theory, the world-volume theory of $N$ M5 branes, compactified on the product of Minkowski $4 d$ space and a genus $g$ Riemann surface with $n$ punctures. Each puncture is associated with a Young diagram specifying the behaviour of the fields at the puncture. Any Riemann surface with punctures can be constructed by gluing spheres with three punctures through tubes. Correspondingly, a generalised quiver theory can be constructed by taking a number of $T_{N}$ theories, the low-energy limit of N M5-branes on a sphere with three punctures, and gauging 
together their flavour symmetries. The $T_{N}$ theory is the building block of generalised quiver theories, it has $S U(N)^{3}$ flavour symmetry, no marginal couplings and does not admit a Lagrangian description. The Gaiotto construction provides a new unifying framework for the study of S-duality of $\mathcal{N}=2$ theories which encodes and extends the early observation by Argyres and Seiberg [2] who discovered the non-Lagrangian E6 theory [3], in strong coupling limit of the $S U(3)$ theory coupled to 6 fundamental hypers.

In this paper we study the $3 \mathrm{~d}$ version of this class of generalised quiver theories. After $S_{1}$ compactification, $4 \mathrm{~d}$ theories flow to an IR point fixed leading to $\mathcal{N}=4$ super-conformal generalised quiver theories in $3 \mathrm{~d}$. The vacuum moduli space of $\mathcal{N}=4$ theories in $3 \mathrm{~d}$ consists of a Coulomb and a Higgs branch corresponding respectively to fluctuations of massless vector multiplets and hypermultiplets. A very interesting duality acting on this moduli space is mirror symmetry [4] which exchanges the Higgs and Coulomb branches of mirror pairs of theories swapping mass parameters for the hypermultiplets with Fayet-Iliopoulos (FI) parameters for the vector multiplets.

In [5], by generalising the construction of mirrors of standard gauge theories involving D3-branes suspended between 5-branes given in [6], it has been found the mirror of generalised quiver theories. For a theory associated to a sphere with $k$ punctures the mirror theory is conjectured to be a star-shaped quiver with $k$ arms coupled to a central $S U(N)$ node. Interestingly star-shaped theories, mirror of generalised quiver theories including $T_{N}$ blocks, turn-out to be always weakly coupled and admit a Lagrangian description. In this paper we will compute partition functions of this class of $3 \mathrm{~d} \mathcal{N}=4$ theories by means of localisation techniques.

The technique of localisation of supersymmetric partition functions involves the addition of a Q-exact operator to the action, which does not affect the path integral, but renders the 1-loop approximation exact. Localisation has been first applied to gauge theories on spheres by Pestun [7], who obtained the partition function of $\mathcal{N}=2$ theories on $S_{4}$. Kapustin, Willett, and Yaakov (KWY) [8] applied localisation techniques to the study of $\mathcal{N}=2$ theories on the three-sphere $S_{3}$. Path integrals reduce to matrix models, which can be solved at large $N$. In particular, the ABJM matrix model, has been solved by Drukker, Mariño, and Putrov [9], who found the famous $N^{3 / 2}$ scaling of the entropy of multiple M2 branes. Chern-Simons matter theories have also been studied $[10,11]$. For an excellent review on this topic and a complete list of references see [12]. Localisation techniques have been extended also to $\mathcal{N}=2$ theories where the anomalous dimensions of the matter fields are not canonical [13, 14].

In [15] localisation has been applied to test non-perturbatively mirror symmetry in strongly coupled super-conformal field theories in three dimensions deformed by real mass terms and FI parameters. For conjectured mirror pairs of theories, partition 
functions, computed by localisation, have been shown to agree provided the mass and FI parameters are exchanged. In [16] Seiberg-like dualities have been tested with similar methods, while in [17], 3d superconformal indices of mirror pairs of theories have been shown to coincide. In this paper we apply the KWY localisation techniques to $3 \mathrm{~d} \mathcal{N}=4$ generalised quiver theories and to their star-shaped mirror dual. One of our main results is the following explicit expression for the partition function of the $T(S U(N))$ quiver tail Fig. 6:

$$
\mathcal{Z}^{T(S U(N))}\left(m_{i} ; e_{j}\right)=\frac{\sum_{\rho \in S^{N}}(-1)^{\rho} e^{2 \pi i \sum_{j}^{N} m_{\rho(j)} e_{j}}}{i^{N(N-1) / 2} \prod_{i<j}^{N} \operatorname{sh}\left(m_{i}-m_{j}\right) \prod_{i<j}^{N} \operatorname{sh}\left(e_{i}-e_{j}\right)},
$$

displaying a manifest self-mirror symmetry under the exchange of mass $m_{i}$ and FI $e_{i}$ parameters. The $T(S U(N))$ quiver tail is the building block to construct generic star shaped quiver theories, by using our exact expression we are able to compute partition functions of this infinite family of theories solving exactly the matrix integrals at finite N. For mirror pairs involving only theories admitting a Lagrangian description we compute partition functions on both sides of the duality and check that they agree provided we exchanged mass and FI parameters. In this way we provide infinite nonperturbative tests of the mirror construction of [5]. Assuming mirror symmetry we then obtain the partition function of the $T_{N}$ theory which is the building block to construct generalised quiver gauge theories.

The papers is organised as follows. In section 2 we introduce the KWY rules for the computation of partition functions on $S_{3}$. We then compute the partition function of the $U(1)$ theory with $N$ flavours which is one of our main tools. In section 3 we study several mirror pairs of rank two models. We begin section 4 with the computation of the partition function of the $T(S U(N))$ theory. We then use this building block to compute partition functions of star shaped quiver theories and compare them with their mirrors. In section 5 we study non-Lagrangian theories. We compute the partition function of the $T_{N}$ theory and use it as a building block to obtain generalised quiver theories. We discuss the TQFT structure of these theories and the associativity of the $T_{N}$ blocks.

\section{Our set-up}

In this section we review the rules for the computation of partition functions on $S_{3}$ and our main tool: the explicit result for the partition function of $U(1)$ theory with $N$ flavours. 


\subsection{The Kapustin-Willet-Yaakov matrix integrals}

Recently it has been shown that the path integral of $3 d$ supersymmetric theories localises to a matrix integral [8]. In the case of $\mathcal{N}=4$ quiver gauge theories, with $S U(N) / U(N)$ gauge groups and fundamental/bifundamental matter, the partition function on $S^{3}$ is given by a matrix integral that is written down using the following rules.

In order to have a slightly more concise notation, we define:

$$
\operatorname{sh}(A) \equiv 2 \sinh (\pi A), \quad \operatorname{ch}(A) \equiv 2 \cosh (\pi A) .
$$

For every gauge group $U(N)$ with FI parameter $\eta$ we have the following integral over the Cartan divided by the residual Weyl symmetry:

$$
\int_{-\infty}^{+\infty} \frac{\mathrm{d}^{N} x}{N !} \prod_{i<j}^{N} s h^{2}\left(x_{i}-x_{j}\right) e^{2 \pi i \eta \sum_{i}^{N} x_{i}} .
$$

For $S U(N)$ gauge groups we replace $d^{N} x$ with $\mathrm{d}^{N} x \delta\left(\sum x_{i}\right)$ and remove the FI parameter.

For every fundamental of mass $m$ attached to the node with integration variables $x_{i}$ we add the factor:

$$
\frac{1}{\prod_{i=1}^{N} \operatorname{ch}\left(x_{i}-m\right)},
$$

while for every bifundamental of mass $m$ attached to the nodes $U\left(N_{1}\right)$ with integration variables $x_{i}$ and $U\left(N_{2}\right)$ with integration variables $y_{i}$ we insert:

$$
\frac{1}{\prod_{i=1}^{N_{1}} \prod_{j=1}^{N_{2}} \operatorname{ch}\left(x_{i}-y_{j}-m\right)} .
$$

Example To see our rules at work we consider the case of a $U(N)$ theory with $K$ flavours with masses $m_{j}$ and FI $\eta$, the corresponding quiver is depicted in Fig. 2.1.

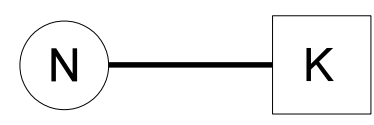

Figure 1. $U(N)$ with $K$ flavours.

The partition function reads:

$$
\hat{\mathcal{Z}}_{K}^{U(N)}=\int \frac{\mathrm{d}^{N} x}{N !} \frac{\prod_{i<j}^{N} \operatorname{sh}^{2}\left(x_{i}-x_{j}\right)}{\prod_{i=1}^{N} \prod_{j=1}^{K} \operatorname{ch}\left(x_{i}-m_{j}\right)} e^{2 \pi i \eta \sum_{i}^{N} x_{i}} .
$$

Here and in the rest of this paper we use the hat to indicate off-shell partition functions. On-shell partition functions satisfy $\sum_{i}^{K} m_{i}=0$. 
The Cauchy determinant formula A tool that is very useful is the so called Cauchy determinant formula:

$$
\frac{\prod_{i<j}^{N} \operatorname{sh}\left(x_{i}-x_{j}\right) \cdot \prod_{i<j}^{N} \operatorname{sh}\left(y_{i}-y_{j}\right)}{\prod_{i, j}^{N} \operatorname{ch}\left(x_{i}-y_{j}\right)}=\sum_{\rho \in S^{N}}(-1)^{\rho} \frac{1}{\prod_{i=1}^{N} \operatorname{ch}\left(x_{i}-y_{\rho(i)}\right)} .
$$

This formula can be used to get rid of the $\prod_{i<j}^{N} s h^{2}\left(x_{i}-x_{j}\right)$ associated to a certain node whenever the number of flavours for that node is twice the number of colours, or greater. That is when that node is a good node, in the Gaiotto-Witten sense [18]. For instance, in the case of $U(N)$ with $K \geq 2 N$ flavours we can separate the flavors into 3 parts, $N+N+(K-2 N)$ with masses $\left\{m_{i}\right\},\left\{\tilde{m}_{i}\right\},\left\{M_{j}\right\}$, and use the Cauchy determinant formula twice, to write $\mathcal{Z}_{K}^{U(N)}$ as

$\frac{\sum_{\rho, \rho^{\prime}}(-1)^{\rho+\rho^{\prime}}}{\prod_{i<j}^{N} \operatorname{sh}\left(m_{i}-m_{j}\right) \operatorname{sh}\left(\tilde{m}_{i}-\tilde{m}_{j}\right)} \int \frac{\mathrm{d}^{N} x}{N !} \frac{e^{2 \pi i \eta \sum_{i}^{N} x_{i}}}{\prod_{i=i}^{N} \operatorname{ch}\left(x_{i}-m_{\rho(i)}\right) \operatorname{ch}\left(x_{i}-\tilde{m}_{\rho^{\prime}(i)}\right) \prod_{j=2 N+1}^{K} \operatorname{ch}\left(x_{i}-M_{j}\right)}$.

We see that the integral factorizes into $N$ single (abelian) integrals and in practice we have to deal with a sum of products of partition functions of $U(1)$ gauge theories. All the Lagrangian $\mathcal{N}=4$ theories that we will study in this paper share this abelianization property, so, to compute exact partition functions, we will need the partition functions of the $U(1)$ theory with $N$ flavours.

\subsection{Abelian integrals: $U(1)$ with $N$ flavours}

In this section we compute the partition functions of the $U(1)$ theory with $N$ flavours.

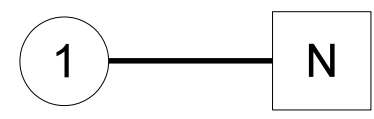

Figure 2. The $U(1)$ theory with $N$ flavours.

The partition function is defined as follows:

$$
\hat{\mathcal{Z}}_{N}^{U(1)}\left(\eta ; m_{i}\right) \equiv \int \mathrm{d} s \frac{e^{2 \pi i s \eta}}{\operatorname{ch}\left(s-m_{1}\right) \operatorname{ch}\left(s-m_{2}\right) \cdots \operatorname{ch}\left(s-m_{N}\right)} .
$$

This is the off-shell partition function of a $U(1)$ gauge theory coupled to $N$ hypers of charge 1 and masses $m_{i}$, which we assume to be real. It is possible to perform the 
integral explicitly using residues, we have to consider $N$ infinite sets of simple poles, located at

$$
s=m_{i}+i\left(2 k_{i}+1\right) / 2 \quad k_{i}=0,1, \ldots
$$

the first set contributes:

$$
\frac{e^{2 \pi i m_{1} \eta}}{\prod_{j \neq 1} \operatorname{ch}\left(m_{1}-m_{j}+i / 2\right)} e^{-\pi \eta} \sum_{k=0}^{\infty}(-1)^{k N} e^{-2 \pi k \eta},
$$

taking into account all the sets of poles we obtain:

$$
\begin{aligned}
\hat{\mathcal{Z}}_{N}^{U(1)}\left(\eta ; m_{i}\right) & =\frac{1}{\left(e^{\pi \eta}-(-1)^{N} e^{-\pi \eta}\right)} \sum_{i=1}^{N} \frac{e^{2 \pi i m_{i} \eta}}{\prod_{j \neq i} \operatorname{ch}\left(m_{i}-m_{j}+i / 2\right)}= \\
& =\frac{1}{i^{N-1}\left(e^{\pi \eta}-(-1)^{N} e^{-\pi \eta}\right)} \sum_{i=1}^{N} \frac{e^{2 \pi i m_{i} \eta}}{\prod_{j \neq i} s h\left(m_{i}-m_{j}\right)} .
\end{aligned}
$$

In the limit of vanishing FI parameter $\eta \rightarrow 0$, we have:

$$
\begin{array}{lll}
\hat{\mathcal{Z}}_{N}^{U(1)}\left(0 ; m_{i}\right) & =\quad 0 \quad \text { odd } N \\
\hat{\mathcal{Z}}_{N}^{U(1)}\left(0 ; m_{i}\right) & =\frac{i}{i^{N-1}} \sum_{i=1}^{N} \frac{m_{i}}{\prod_{j \neq i} \operatorname{sh}\left(m_{i}-m_{j}\right)} &
\end{array}
$$

Example $N=1$ :

In the special case of one flavour we get :

$$
\hat{\mathcal{Z}}_{1}^{U(1)}(\eta, m)=\int \mathrm{d} a \frac{e^{2 \pi i a \eta}}{c h(a-m)}=\frac{e^{2 \pi i m \eta}}{c h(\eta)} .
$$

On shell, for $m=0$ we have:

$$
\mathcal{Z}_{1}^{U(1)}(\eta)=\frac{1}{\operatorname{ch}(\eta)}=\mathcal{Z}_{1 \text { freeH }}(\eta)
$$

which is a manifestation of the basic statement of abelian mirror symmetry [4]: the $\mathcal{N}=4, U(1)$ theory with one flavour and with FI parameter $\eta$, is mirror of the $\mathcal{N}=4$ theory of 1 free hyper with mass $\eta$.

Example $N=2$ :

In the special case of 2 flavours we get:

$$
\left.\mathcal{Z}_{2}^{U(1)}(\eta, m)=\int \mathrm{d} s \frac{e^{2 \pi i s \eta}}{\operatorname{ch}(s-m / 2) \operatorname{ch}(s+m / 2)}=\frac{-i\left(e^{\pi i m \eta}-e^{-\pi i m \eta}\right)}{\operatorname{sh}(m / 2+m / 2) s(\eta)}=\frac{2 \sin (\pi \eta m)}{\operatorname{sh}(m) \operatorname{sh}(\eta)}, 16\right)
$$

we see that the partition function is symmetric in $m \leftrightarrow \eta$ :

$$
\mathcal{Z}_{2}^{U(1)}(\eta, m)=\mathcal{Z}_{2}^{U(1)}(m, \eta) .
$$

This is a manifestation of the fact that $U(1)$ with 2 flavours is self-mirror [4]. 


\section{$3 \quad U(2)$ and $S U(2)$ models}

In this section we compute partition functions of the following quiver gauge theories:

1. SU(2) with K flavours.

2. $U(2)^{k+1} \times U(1)^{4} / / U(1)$, the $D_{k}$ quiver.

3. $S U(2) \times U(1)^{N}$ star-shaped.

We will compute the partition functions of these models as functions of FI and mass parameters. Some of these theories are related by mirror symmetry as explained in the following table where we indicate the dimensions of Higgs and Coulomb branches and the number of masses and FI parameters:

\begin{tabular}{|c|c|c|c|c|}
\hline Model & dim Higgs & dim Coulomb & \# masses & \# of FI's \\
\hline \hline $\mathrm{SU}(2)$ with K flavors & $2 K-3$ & 1 & $K$ & 0 \\
\hline$S U(2) \times U(1)^{N}$ star-shape & $N-3$ & $N+1$ & 0 & $N$ \\
\hline$S U(2)^{k}$ linear-shape & $k+4$ & $k$ & $k+3$ & 0 \\
\hline$U(2)^{k+1} \times U(1)^{4} / / U(1) D_{k}$-shape & 1 & $2 k+5$ & 0 & $k+4$ \\
\hline
\end{tabular}

One can easily single out mirror pairs as pairs of theories for which the Higgs and Coulomb branches are exchanged.

\section{$3.1 S U(2)$ with K flavours}

The partition function with quiver diagram in Fig. $3^{1}$ is defined as

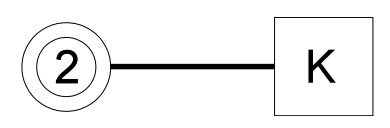

Figure 3. $S U(2)$ with $K$ flavours.

$$
\hat{\mathcal{Z}}_{K}^{S U(2)}\left(m_{i}\right)=\int \frac{\mathrm{d} x_{1} \mathrm{~d} x_{2}}{2 !} \delta\left(x_{1}+x_{2}\right) \frac{\operatorname{sh}^{2}\left(x_{1}-x_{2}\right)}{\prod_{j=1}^{K} \operatorname{ch}\left(x_{1}-m_{j}\right) \operatorname{ch}\left(x_{2}-m_{j}\right)} .
$$

\footnotetext{
${ }^{1}$ We use a double circle, as opposed to a simple one to denote $S U(N)$ nodes.
} 
We separate the $K \geq 4$ flavors into $2+2+(K-4)$ so we can use the Cauchy determinant formula twice and get rid of the $\operatorname{sh}^{2}\left(x_{1}-x_{2}\right)$ numerator:

$$
\begin{aligned}
& \frac{1}{\operatorname{sh}\left(m_{1}-m_{2}\right) \operatorname{sh}\left(m_{3}-m_{4}\right)} \int \frac{\mathrm{d} x}{\prod_{p=5}^{K} \operatorname{ch}\left(x-m_{p}\right) \operatorname{ch}\left(x+m_{p}\right)} \times \\
& \times\left(\frac{1}{\operatorname{ch}\left(x-m_{1}\right) \operatorname{ch}\left(x+m_{2}\right)}-\frac{1}{\operatorname{ch}\left(x-m_{2}\right) \operatorname{ch}\left(x+m_{1}\right)}\right) \frac{1}{\operatorname{ch}\left(x-m_{3}\right) \operatorname{ch}\left(x+m_{4}\right)} .
\end{aligned}
$$

Now we can perform the last integral over $x$ using eq. (2.11) with $2(K-4)+4=2 K-4$ flavours and vanishing FI parameter. Let us focus on the terms proportional to $m_{1}$, there are 2 such terms and they contribute as

$$
\begin{gathered}
+\frac{m_{1}}{\prod_{i \geq 5} \operatorname{sh}\left(m_{1}-m_{i}\right) \operatorname{sh}\left(m_{1}+m_{i}\right) \operatorname{sh}\left(m_{1}+m_{2}\right) \operatorname{sh}\left(m_{1}-m_{3}\right) \operatorname{sh}\left(m_{1}+m_{4}\right)} \\
-\frac{-m_{1}}{\prod_{i \geq 5} \operatorname{sh}\left(m_{1}-m_{i}\right) \operatorname{sh}\left(m_{1}+m_{i}\right) \operatorname{sh}\left(-m_{1}-m_{2}\right) \operatorname{sh}\left(-m_{1}-m_{3}\right) \operatorname{sh}\left(-m_{1}+m_{4}\right)}
\end{gathered}
$$

summing these two terms we get

$$
\frac{m_{1} s h\left(2 m_{1}\right)}{\prod_{j \neq 1}\left(s^{2}\left(m_{1}\right)-s h^{2}\left(m_{j}\right)\right)} .
$$

By symmetry in the $m_{i}$, we obtain the following exact expression for the partition function:

$$
\hat{\mathcal{Z}}_{K}^{S U(2)}\left(m_{i}\right)=\sum_{i=1}^{K} \frac{m_{i} \operatorname{sh}\left(2 m_{i}\right)}{\prod_{j \neq i}\left(\operatorname{sh}^{2}\left(m_{i}\right)-s^{2}\left(m_{j}\right)\right)} .
$$

\subsection{The $D_{k}$ quiver}

In this section we study the $U(2)^{k+1} \times U(1)^{4} / / U(1)$ theory also known as the $D_{k}$ quiver. As in Fig. 4 we denote by $\xi_{I}$ the FI's and by $z_{i}^{(I)}$ the Cartan's of the $U(2)$ nodes with $I=1, \cdots, k, i=1,2$. We then denote by $x_{i}$ the Cartan's of the $S U(2)$ node and by $\eta_{a, b, c, d}$, $a, b, c, d$ the FI's and Cartan's of the $U(1)$ nodes. 


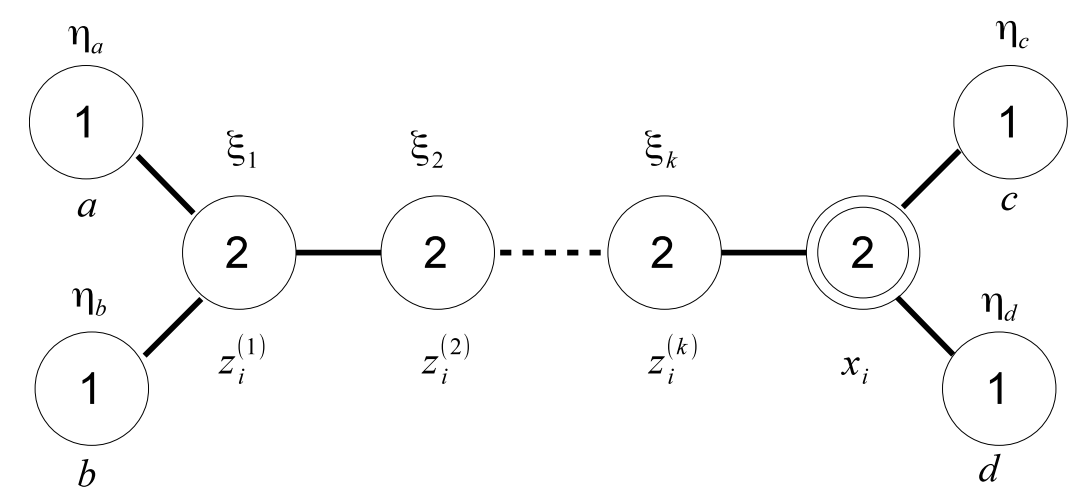

Figure 4. the $D_{k}$ quiver

The partition function is given by:

$$
\begin{aligned}
& \mathcal{Z}_{D_{k}}=\frac{1}{2^{k+1}} \int \mathrm{d} a \mathrm{~d} b \mathrm{~d} c \mathrm{~d} d \prod_{I}^{k} \mathrm{~d} z_{1}^{I} \mathrm{~d} z_{2}^{I} \mathrm{~d} x_{1} \mathrm{~d} x_{2} \delta\left(x_{1}+x_{2}\right) \frac{e^{2 \pi i \sum_{I} \xi_{I}\left(z_{1}^{I}+z_{2}^{I}\right)} e^{2 \pi i\left(\eta_{a} a+\eta_{b} b+\eta_{c} c+\eta_{d} d\right)}}{\prod_{i}^{2} \operatorname{ch}\left(z_{i}^{(1)}-a\right) \operatorname{ch}\left(z_{i}^{(1)}-b\right) \operatorname{ch}\left(x_{i}-c\right) \operatorname{ch}\left(x_{i}-d\right)} \\
& \times \frac{\operatorname{sh}\left(z_{1}^{(1)}-z_{2}^{(1)}\right)^{2} \operatorname{sh}\left(z_{1}^{(2)}-z_{2}^{(2)}\right)^{2} \cdots \operatorname{sh}\left(z_{1}^{(k)}-z_{2}^{(k)}\right)^{2} \operatorname{sh}\left(x_{1}-x_{2}\right)^{2}}{\prod_{i, j}^{2} \operatorname{ch}\left(z_{i}^{(2)}-z_{j}^{(1)}\right) \cdots \operatorname{ch}\left(z_{i}^{(k)}-z_{j}^{(k-1)}\right) \operatorname{ch}\left(x_{i}-z_{j}^{(k)}\right)}= \\
& =\frac{1}{2^{k+1}} \frac{1}{s h \eta_{a} s h \eta_{c}} \int \mathrm{d} b \mathrm{~d} d \prod_{I}^{k} \mathrm{~d} z_{1}^{I} \mathrm{~d} z_{2}^{I} \mathrm{~d} x_{1} \mathrm{~d} x_{2} \delta\left(x_{1}+x_{2}\right) \frac{e^{2 \pi i \sum_{I} \xi_{I}\left(z_{1}^{I}+z_{2}^{I}\right)} e^{2 \pi i\left(\eta_{b} b+\eta_{d} d\right)}}{\prod_{i}^{2} \operatorname{ch}\left(z_{i}^{(1)}-b\right) \operatorname{ch}\left(x_{i}-d\right)} \\
& \times\left(e^{2 \pi i \eta_{a} z_{1}^{(1)}}-e^{2 \pi i \eta_{a} z_{2}^{(1)}}\right)\left(e^{2 \pi i \eta_{c} x_{1}}-e^{2 \pi i \eta_{c} x_{2}}\right)
\end{aligned}
$$

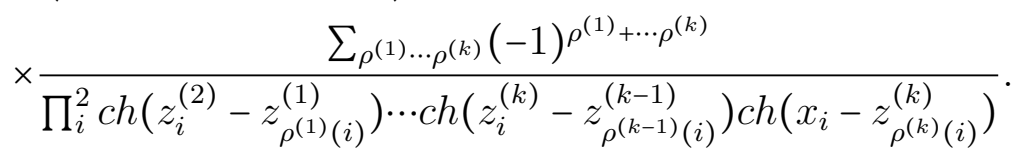

By reordering the integration variables $z_{i}^{I}$ we get rid of $k$ sums over permutations and gain a factor $2^{k}$. It is now convenient to take the (inverse) Fourier transform of each ch to obtain: 


$$
\begin{aligned}
\mathcal{Z}_{D_{k}}= & \frac{1}{2} \frac{1}{s h \eta_{a} s h \eta_{c}} \int \mathrm{d} b \mathrm{~d} d \prod_{I}^{k} d z_{1}^{I} d z_{2}^{I} \mathrm{~d} x_{1} \mathrm{~d} x_{2} \delta\left(x_{1}+x_{2}\right) e^{2 \pi i \sum_{I} \xi_{I}\left(z_{1}^{I}+z_{2}^{I}\right)} e^{2 \pi i\left(\eta_{b} b+\eta_{d} d\right)} \\
& \times\left(e^{2 \pi i \eta_{a} z_{1}^{(1)}}-e^{2 \pi i \eta_{a} z_{2}^{(1)}}\right)\left(e^{2 \pi i \eta_{c} x_{1}}-e^{2 \pi i \eta_{c} x_{2}}\right) \\
& \times \int \frac{\mathrm{d} s_{1} \mathrm{~d} s_{2}}{\operatorname{ch} s_{1} c h s_{2}} e^{2 \pi i\left(s_{1}\left(z_{1}^{(1)}-b\right)+s_{2}\left(z_{2}^{(1)}-b\right)\right)} \int \frac{\mathrm{d} p_{1} \mathrm{~d} p_{2}}{\operatorname{ch} p_{1} \operatorname{ch} p_{2}} e^{2 \pi i\left(p_{1}\left(x_{1}-d\right)+p_{2}\left(x_{2}-d\right)\right)} \\
& \times \int \frac{\prod_{I=2}^{k+1} \mathrm{~d} t_{1}^{(I)} \mathrm{d} t_{2}^{(I)}}{c h t_{1}^{(I)}} \operatorname{cht}_{2}^{(I)} e^{2 \pi i \sum_{I=2}^{k}\left(t_{1}^{(I)}\left(z_{1}^{(I)}-z_{1}^{(I-1)}\right)+t_{2}^{(I)}\left(z_{2}^{(I)}-z_{2}^{(I-1)}\right)\right)} e^{2 \pi i\left(t_{1}^{(k+1)}\left(x_{1}-z_{1}^{(k)}\right)+t_{2}^{(k+1)}\left(x_{2}-z_{2}^{(k)}\right)\right)} .
\end{aligned}
$$

We will now show that the above expression coincides with the partition function of the mirror theory, the $S U(2)$ theory with $K=3+k$ flavours (Fig. 3), provided we used the following dictionary:

$$
\begin{gathered}
\eta_{a}=m_{4}-m_{3}, \quad \eta_{b}=m_{3}+m_{4}, \quad \eta_{c}=m_{2}-m_{1}, \quad \eta_{d}=m_{1}+m_{2} \\
\xi_{I}=m_{4+I}-m_{3+I}, \quad I=1, \cdots k .
\end{gathered}
$$

There are four terms in eq. (3.5). Let's consider first the term proportional to $e^{2 \pi i \eta_{a} z_{1}^{(1)}} e^{2 \pi i \eta_{c} x_{1}}$, the integration over $b, d, x_{i}, z_{i}^{I}$ produces the following deltas:

$$
\begin{aligned}
& \delta\left(s_{1}-t_{1}^{(1)}+\xi_{1}+\eta_{a}\right), \quad \delta\left(s_{2}-t_{2}^{(1)}+\xi_{1}\right), \\
& \delta\left(t_{1}^{(I-1)}-t_{1}^{(I)}+\xi_{I}\right) \quad \delta\left(t_{2}^{(I-1)}-t_{2}^{(I)}+\xi_{I}\right), \quad I=2, \cdots k+1, \\
& \delta\left(-s_{1}-s_{2}+\eta_{b}\right), \quad \delta\left(-p_{1}-p_{2}+\eta_{d}\right), \\
& \delta\left(p_{1}-p_{2}+t_{1}^{(k+1)}-t_{2}^{(k+1)}+\eta_{c}\right),
\end{aligned}
$$

solving for $s_{1}$ and expressing the result in terms of $x=s_{1}+m_{3}$ we obtain the following combination of $c h^{\prime} s$ in the denominator:

$$
\begin{aligned}
& \operatorname{ch}\left(x+m_{3}\right) \operatorname{ch}\left(x-m_{4}\right) \operatorname{ch}\left(x+m_{5}\right) \operatorname{ch}\left(x-m_{5}\right) \cdots \operatorname{ch}\left(x+m_{k+4}\right) \operatorname{ch}\left(x-m_{k+4}\right) \\
& \operatorname{ch}\left(x+m_{2}\right) \operatorname{ch}\left(x-m_{1}\right) .
\end{aligned}
$$

The remaining three terms give similar contributions. Putting everything together we rewrite the partition function as:

$$
\begin{aligned}
\mathcal{Z}_{D_{k}}= & \frac{1}{\operatorname{sh}\left(m_{2}-m_{1}\right) \operatorname{sh}\left(m_{4}-m_{3}\right)} \int \mathrm{d} x \frac{1}{\prod_{I}^{k} \operatorname{ch}\left(x+m_{4+I}\right) \operatorname{ch}\left(x-m_{4+I}\right)} \\
& \times\left(\frac{1}{\operatorname{ch}\left(x-m_{1}\right) \operatorname{ch}\left(x+m_{2}\right)}-\frac{1}{\operatorname{ch}\left(x+m_{1}\right) \operatorname{ch}\left(x-m_{2}\right)}\right) \frac{1}{\operatorname{ch}\left(x-m_{3}\right) \operatorname{ch}\left(x+m_{4}\right)} .
\end{aligned}
$$


This expression coincides with the partition function of the $S U(2)$ theory with $K=3+k$ flavours, eq. (3.1).

\section{3 $S U(2) \times U(1)^{N}$ star shaped}

Let's consider now the $S U(2) \times U(1)^{N}$ star shaped theory depicted in Fig. 5, with FI parameters $\eta_{i}, i=1, \cdots N$.

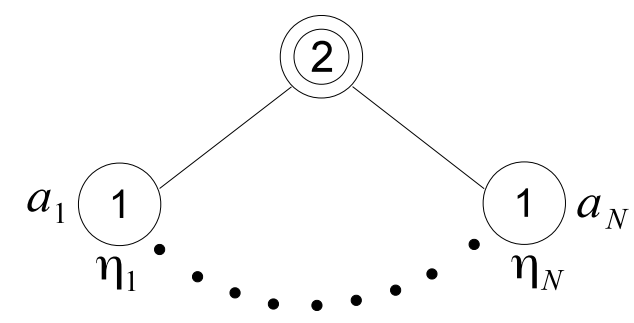

Figure 5. $S U(2) \times U(1)^{N}$.

The partition function reads:

$$
\mathcal{Z}_{N}\left(\eta_{i}\right)=\int \prod_{i}^{N} d a_{i} \frac{\mathrm{d} x_{1} \mathrm{~d} x_{2}}{2 !} \delta\left(x_{1}+x_{2}\right) e^{2 \pi i \sum_{i} \eta_{i} a_{i}} \frac{\operatorname{sh}^{2}\left(x_{1}-x_{2}\right)}{\prod_{i=1}^{2} \prod_{j=1}^{N} \operatorname{ch}\left(x_{i}-a_{j}\right)} .
$$

The Abelian integrals over $a_{i}$ can be performed using the result in (2.16) and we obtain:

$$
\begin{aligned}
\mathcal{Z}_{N}\left(\eta_{i}\right) & =\frac{1}{2} \int \mathrm{d} x_{1} \mathrm{~d} x_{2} \delta\left(x_{1}+x_{2}\right) \prod_{i} \hat{\mathcal{Z}}_{2}^{U(1)}\left(\eta_{i} ; x_{1}, x_{2}\right) \\
& =\frac{1}{2} \int \frac{\mathrm{d} x}{\operatorname{sh}(2 x)^{N-2}} \prod_{i=1}^{N} \frac{2 \sin \left(2 \pi \eta_{i} x\right)}{\operatorname{sh}\left(\eta_{i}\right)} .
\end{aligned}
$$

The integral is convergent for $N \geq 3$. For $N<3$ we have bad theories in the GaiottoWitten [18] sense while $N=3$ corresponds to an ugly theory mirror of the theory of 4 free hypers. For $N>3$ the mirror is a linear quiver with $N-3 \mathrm{SU}(2)$ gauge groups, a good theory. In eq. (3.11) the $S_{N}$ symmetry among the $N$ FI parameters is explicit, and the last integral can be performed in terms of the sin / cos transform of $\sinh (t)^{-k}$, after expanding the product of the $N \sin (\eta x)^{\prime}$ 's in terms of sums of $\sin / \cos$ functions. We will perform the integral for the cases $N=3$ and $N=4$.

More results for generic $S U(2)$ star shaped quivers can be obtained as particular cases of the results we give in section 4.3. 
Example $S U(2) \times U(1)^{3}$ :

In order to perform the computation of $\mathcal{Z}_{3}$ we use the trigonometric identity $2 \sin (a) 2 \sin (b) 2 \sin (c)=2(\sin (a+b+c)+\sin (a-b-c)+\sin (-a+b-c)+\sin (-a-b+c))$ and the sin-transform:

$$
\int \mathrm{d} s \frac{\sin (2 \pi \eta s)}{\operatorname{sh}(2 s)}=\frac{\operatorname{sh}(\eta / 2)}{2 \operatorname{ch}(\eta / 2)}
$$

to get

$$
\mathcal{Z}_{3}=\frac{\operatorname{th}\left(\left(\eta_{1}+\eta_{2}+\eta_{3}\right) / 2\right)+\operatorname{th}\left(\left(\eta_{1}-\eta_{2}-\eta_{3}\right) / 2\right)+\operatorname{th}\left(\left(-\eta_{1}+\eta_{2}-\eta_{3}\right) / 2\right)+\operatorname{th}\left(\left(-\eta_{1}-\eta_{2}+\eta_{3}\right) / 2\right)}{2 \operatorname{sh}\left(\eta_{1}\right) \operatorname{sh}\left(\eta_{2}\right) \operatorname{sh}\left(\eta_{3}\right)} .
$$

After few manipulation the above expression simplifies to2

$$
\mathcal{Z}_{3}=\frac{1}{2 \operatorname{ch}\left(\left(\eta_{1}+\eta_{2}+\eta_{3}\right) / 2\right) \operatorname{ch}\left(\left(\eta_{1}-\eta_{2}-\eta_{3}\right) / 2\right) \operatorname{ch}\left(\left(-\eta_{1}+\eta_{2}-\eta_{3}\right) / 2\right) \operatorname{ch}\left(\left(-\eta_{1}-\eta_{2}+\eta_{3}\right) / 2\right)} .
$$

This is the partition function of 4 free hypers with masses $\left(\eta_{1} \pm \eta_{2} \pm \eta_{3}\right) / 2$.

Example $S U(2) \times U(1)^{4}$ :

In the special case $N=4$ we need to use a trigonometric identity to express the product of $4 \sin (x)$ 's in terms of the sum of $8 \cos (x)$ 's:

$$
\begin{aligned}
& \frac{1}{2} \int \frac{\mathrm{d} x}{\operatorname{sh}(2 x)^{2}} \prod_{j=1}^{4} 2 \sin \left(2 \pi \eta_{i} x\right)=\int \frac{\mathrm{d} x}{\operatorname{sh}(2 x)^{2}}\left(\cos \left(2 \pi\left(\eta_{1}+\eta_{2}+\eta_{3}+\eta_{4}\right) x\right)+\cos \left(2 \pi\left(\eta_{1}+\eta_{2}-\eta_{3}-\eta_{4}\right) x\right)+\right. \\
& \quad+\cos \left(2 \pi\left(-\eta_{1}+\eta_{2}+\eta_{3}-\eta_{4}\right) x\right)+\cos \left(2 \pi\left(\eta_{1}-\eta_{2}+\eta_{3}-\eta_{4}\right) x\right)-\cos \left(2 \pi\left(-\eta_{1}+\eta_{2}+\eta_{3}+\eta_{4}\right) x\right)- \\
& \left.\quad-\cos \left(2 \pi\left(\eta_{1}-\eta_{2}+\eta_{3}+\eta_{4}\right) x\right)-\cos \left(2 \pi\left(\eta_{1}+\eta_{2}-\eta_{3}+\eta_{4}\right) x\right)-\cos \left(2 \pi\left(\eta_{1}+\eta_{2}+\eta_{3}-\eta_{4}\right) x\right)\right)
\end{aligned}
$$

and use the following cos-transform:

$$
\int \mathrm{d} x \frac{\cos (2 \pi \xi x)}{\operatorname{sh}(2 x)^{2}}=\frac{\xi \operatorname{ch}(\xi / 2)}{2 \operatorname{sh}(\xi / 2)}
$$

to compute the last integral.

At this point we change variables with the dictionary:

$$
\eta_{1}=\tilde{m}_{1}-\tilde{m}_{2}, \quad \eta_{2}=\tilde{m}_{1}+\tilde{m}_{2}, \quad \eta_{3}=m_{1}-m_{2}, \quad \eta_{4}=m_{1}+m_{2} .
$$

\footnotetext{
${ }^{2}$ This expression has been previously obtained in [19].
} 
We now collects terms proportional to $m_{1}$ :

$$
\frac{m_{1}\left(\frac{\operatorname{ch}\left(m_{1}+\tilde{m}_{1}\right)}{\operatorname{sh}\left(m_{1}+\tilde{m}_{1}\right)}+\frac{\operatorname{ch}\left(m_{1}-\tilde{m}_{1}\right)}{\operatorname{sh}\left(m_{1}-\tilde{m}_{1}\right)}-\frac{\operatorname{ch}\left(m_{1}-\tilde{m}_{2}\right)}{\operatorname{sh}\left(m_{1}-\tilde{m}_{2}\right)}-\frac{\operatorname{ch}\left(m_{1}+\tilde{m}_{2}\right)}{\operatorname{sh}\left(m_{1}+\tilde{m}_{2}\right)}\right)}{\operatorname{sh}\left(m_{1}-m_{2}\right) \operatorname{sh}\left(m_{1}+m_{2}\right) \operatorname{sh}\left(\tilde{m}_{1}-\tilde{m}_{2}\right) \operatorname{sh}\left(\tilde{m}_{1}+\tilde{m}_{2}\right)} .
$$

We get similar expressions for the other $m$ 's. After few manipulations, putting all together we obtain ${ }^{3}$ :

$$
\mathcal{Z}^{S U(2) \times U(1)^{4}}\left(m_{i}\right)=\sum_{i=1}^{4} \frac{m_{i} \operatorname{sh}\left(2 m_{i}\right)}{\prod_{j \neq i}\left(s h^{2}\left(m_{i}\right)-s h^{2}\left(m_{j}\right)\right)} .
$$

This is precisely the partition function of the $S U(2)$ theory with 4 flavours given in eq. (3.3).

\section{Lagrangian theories}

We will now move to the study of generalised quiver theories corresponding to spheres with two generic punctures and any number of simple ones. These theories admit a Lagrangian description. We will compute explicitly their partition functions and those of their mirror pairs, which are star shaped quiver theories. We start with the explicit evaluation of the partition function of the $T(S U(N))$ quiver theory which, being the mirror of a full puncture, is the main building block.

\section{$4.1 T(S U(N))$}

In this section we will compute the partition function of the $T(S U(N))$ quiver theory depicted in Fig. 6. $T(G)$ is a $3 d \mathcal{N}=4$ gauge theory at the IR super-conformal fixed point, with global symmetry $G \times G^{L}\left(G^{L}\right.$ is the Langlands dual of $\left.G\right)$. The Higgs and Coulomb branches are respectively acted by $G$ and $G^{L}$. Under the $\mathcal{N}=4$ mirror transformation, $T(G)$ is mapped to $T\left(G^{L}\right)$. In the $G=S U(N)$ case the Coulomb and Higgs branches are isomorphic and FI and mass parameters are exchanged by mirror symmetry.

$T(S U(N))$ will be our fundamental building block to compute the partition function of generic star shaped quivers corresponding to spheres with generic punctures. Indeed, in [5] the quiver tail $T(S U(N))$ has been identified with the mirror of the full puncture $\odot$, we will then equivalently denote the $T(S U(N))$ partition function as $\mathcal{Z}^{T(S U(N))}$ or as $\mathcal{Z}_{N}^{\odot}$.

Let's fix the notation as in Fig. 6. Let $\eta_{d}, d=1, \cdots N-1$ be the FI parameters and $x_{i}^{(d)}$ (with $\left.i=1, \cdots d\right)$ the Cartan's of the $U(1) \times \cdots \times U(N-1)$ nodes. Let also $m_{i}, i=1, \cdots N, \sum_{i}^{N} m_{i}=0$ be the masses acted by the $S U(N)$ flavour symmetry. The

\footnotetext{
${ }^{3}$ Set $m_{3}=\tilde{m}_{1}$ and $m_{4}=\tilde{m}_{2}$.
} 


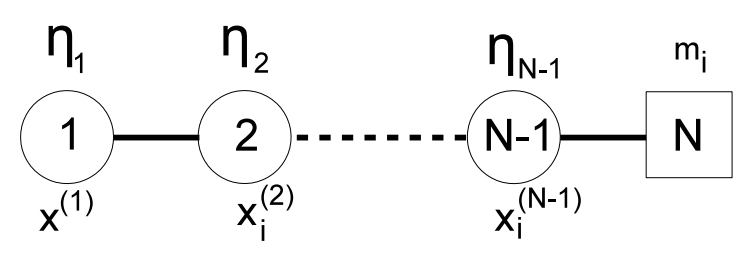

Figure 6. The quiver diagram for $T(S U(N))$

off-shell $T(S U(N))$ partition function is given, by the KWY rules, by:

$$
\begin{gathered}
\hat{\mathcal{Z}}^{T(S U(N))}\left(m_{1}, \ldots, m_{N} ; \eta_{1}, \ldots, \eta_{N-1}\right)=\int \mathrm{d} x^{(1)} \times \frac{\mathrm{d} x_{1}^{(2)} \mathrm{d} x_{2}^{(2)}}{2 !} \cdots \times \prod_{i}^{N-1} \frac{\mathrm{d} x_{i}^{(N-1)}}{(N-1) !} \times \\
\times e^{2 \pi i\left(\eta_{1} x^{(1)}+\eta_{2}\left(x_{1}^{(2)}+x_{2}^{(2)}\right)+\cdots+\eta_{N-1}\left(x_{1}^{(N-1)}+\cdots+x_{N-1}^{(N-1)}\right)\right)} \times \\
\times \frac{\prod_{d=2}^{N-1} \prod_{i<j}^{d} \operatorname{sh}^{2}\left(x_{i}^{(d)}-x_{j}^{(d)}\right)}{\prod_{i=1}^{N} \prod_{j}^{N-1} \operatorname{ch}\left(m_{i}-x_{j}^{(N-1)}\right) \cdots \prod_{i}^{d} \prod_{j=1}^{d-1} \operatorname{ch}\left(x_{i}^{(d)}-x_{j}^{(d-1)}\right) \cdots \prod_{j=1}^{2} \operatorname{ch}\left(x_{j}^{(2)}-x^{(1)}\right)} .
\end{gathered}
$$

It is convenient to change variables for the FI parameters, from $\eta_{i}$ to $e_{i}$ :

$$
\eta_{i}=e_{i}-e_{i+1}, \quad \text { for } \quad i=1, \ldots, N-1 \quad \text { with } \quad \sum_{i}^{N} e_{i}=0 .
$$

We claim that the result of the integral (4.1) is:

$$
\hat{\mathcal{Z}}^{T(S U(N))}\left(m_{i} ; e_{j}\right)=\frac{\sum_{\rho \in S^{N}}(-1)^{\rho} e^{2 \pi i \sum_{j}^{N} m_{\rho(j)}\left(e_{j}-e_{N}\right)}}{i^{N(N-1) / 2} \prod_{i<j}^{N} \operatorname{sh}\left(m_{i}-m_{j}\right) \prod_{i<j}^{N} \operatorname{sh}\left(e_{i}-e_{j}\right)} .
$$

This is one of our main results. We will provide a proof of this formula by induction at the end of this section.

In terms of the variables $\eta_{i}, i=1, \ldots, N-1$, the formula is a bit more complicated:

$$
\hat{\mathcal{Z}}^{T(S U(N))}\left(m_{i} ; \eta_{i}\right)=\frac{\sum_{\rho \in S^{N}}(-1)^{\rho} e^{2 \pi i \sum_{i=1}^{N-1} m_{\rho(i)}\left(\eta_{N-1}+\cdots+\eta_{i}\right)}}{i^{N(N-1) / 2} \prod_{i<j}^{N} \operatorname{sh}\left(m_{i}-m_{j}\right) \prod_{d=1}^{N-1} \prod_{k=1}^{d} \operatorname{sh}\left(\eta_{d}+\eta_{d-1}+\cdots+\eta_{k}\right)}
$$

Let us check what happens for $N=2$, where $T(S U(2))$ is simply the $U(1)$ theory with 2 flavours. In this case eq. (4.3) reduces as expected to:

$$
\hat{\mathcal{Z}}^{T(S U(2))}\left(m_{1}, m_{2} ; \eta\right)=\frac{e^{2 \pi i m_{1} \eta}-e^{2 \pi i m_{2} \eta}}{i s h\left(m_{1}-m_{2}\right) \operatorname{sh}(\eta)}=\hat{Z}_{2}^{U(1)}\left(m_{1}, m_{2} ; \eta\right)
$$


Check of the self-mirror property: The expected self-mirror property of the $T(S U(N))$ theory, which exchanges $m_{i} \leftrightarrow e_{i}$, is manifest in our explicit expression for the partition function eq. (4.3). By using that:

$$
\sum_{\rho \in S^{N}}(-1)^{\rho} e^{2 \pi i \sum_{j} m_{\rho(j)} e_{j}}=\sum_{\rho^{\prime} \in S^{N}}(-1)^{\rho^{\prime}} e^{2 \pi i \sum_{j} e_{\rho^{\prime}(j)} m_{j}}
$$

it is clear that the on-shell partition function $\left(\sum_{i}^{N} m_{i}=0\right)$ is self-mirror:

$$
\mathcal{Z}^{T(S U(N))}\left(m_{i} ; e_{j}\right)=\mathcal{Z}^{T(S U(N))}\left(e_{i} ; m_{j}\right)
$$

\subsubsection{Proof of the formula by induction}

To prove our expression for $\hat{\mathcal{Z}}^{T(S U(N))}$ we use it as a building block to construct $\hat{\mathcal{Z}}^{T(S U(N+1))}$; we gauge the flavour symmetry multiplying by the $\prod_{i<j}^{N} s h^{2}\left(x_{i}-x_{j}\right)$ and integrating over the $S U(N)$ Cartan. We then add the FI parameter $\eta_{N}$ and $N+1$ fundamentals of masses $m_{i}, i=1, \cdots, N+1$ :

$$
\hat{\mathcal{Z}}^{T(S U(N+1))}=\int \frac{\mathrm{d}^{N} x}{N !} \prod_{i<j}^{N} s h^{2}\left(x_{i}-x_{j}\right) \frac{\hat{\mathcal{Z}}^{T(S U(N))}\left(x ; \eta_{1} \ldots \eta_{N-1}\right)}{\prod_{i=1}^{N} \prod_{j=1}^{N+1} \operatorname{ch}\left(x_{i}-m_{j}\right)} e^{2 \pi i \eta_{N} \sum_{i}^{N} x_{i}}
$$

Now we separate the $N+1$ fundamentals in a group of $N$ masses $m_{i}, i=1, \cdots N$ plus a singlet $m_{N+1}$ and plug our result for $\hat{\mathcal{Z}}^{T(S U(N)}$ to get:

$$
\begin{aligned}
\hat{\mathcal{Z}}^{T(S U(N+1))}= & \frac{1}{\prod_{i<j}^{N} \operatorname{sh}\left(e_{i}-e_{j}\right)} \int \frac{\mathrm{d}^{N} x}{N !} e^{-2 \pi i e_{N} \sum_{k}^{N} x_{k}} \sum_{\rho \in S^{N}}(-1)^{\rho} e^{2 \pi i \sum_{j}^{N} e_{\rho(j)} x_{j}} e^{2 \pi i \eta_{N} \sum_{i}^{N} x_{i}} \\
& \times \frac{\prod_{i<j}^{N} \operatorname{sh}\left(x_{i}-x_{j}\right)}{i^{N(N-1) / 2} \prod_{i, j=1}^{N} \operatorname{ch}\left(x_{i}-m_{j}\right) \prod_{i=1}^{N} \operatorname{ch}\left(x_{i}-m_{N+1}\right)}
\end{aligned}
$$

we now use the Cauchy determinant formula once

$$
\frac{1}{\prod_{p<q}^{N} \operatorname{sh}\left(m_{p}-m_{q}\right) \prod_{i<j}^{N} \operatorname{sh}\left(e_{i}-e_{j}\right)} \sum_{\rho, \rho^{\prime} \in S^{N}}(-1)^{\rho+\rho^{\prime}} \int \frac{\mathrm{d}^{N} x}{N !} \frac{e^{-2 \pi i e_{N} \sum_{i}^{N} x_{i}} e^{2 \pi i \sum_{j}^{N} e_{\rho(j)} x_{j}} e^{2 \pi i \eta_{N} \sum_{i}^{N} x_{i}}}{i^{N(N-1) / 2} \prod_{i}^{N} \operatorname{ch}\left(x_{i}-m_{N+1}\right) \operatorname{ch}\left(x_{i}-m_{\rho^{\prime}(i)}\right)},
$$

we then reorder the integration variables $x_{i}$ we get rid of one sum over permutations and cancel the factor $N$ ! and get:

$\frac{1}{\prod_{p<q}^{N} \operatorname{sh}\left(m_{p}-m_{q}\right) \prod_{i<j}^{N} \operatorname{sh}\left(e_{i}-e_{j}\right)} \sum_{\rho \in S^{N}}(-1)^{\rho} \int d^{N} x \frac{e^{2 \pi i \sum_{j}^{N} x_{j}\left(e_{j}-e_{N+1}\right)}}{i^{N(N-1) / 2} \prod_{i}^{N} \operatorname{ch}\left(x_{i}-m_{N+1}\right) \operatorname{ch}\left(x_{i}-m_{\rho(i)}\right)}$, 
where we introduced the new variable $e_{N+1}$ by $\eta_{N} \equiv e_{N}-e_{N+1}$. The integral at this point factorizes into abelian 2-flavors integrals:

$$
\begin{aligned}
& \frac{1}{i^{N(N-1) / 2} \prod_{p<q}^{N} \operatorname{sh}\left(m_{p}-m_{q}\right) \prod_{i<j}^{N} \operatorname{sh}\left(e_{i}-e_{j}\right)} \sum_{\rho \in S^{N}}(-1)^{\rho} \prod_{i=1}^{N} \frac{\left(e^{2 \pi i m_{\rho(i)}\left(e_{j}-e_{N+1}\right)}-e^{2 \pi i m_{N+1}\left(e_{i}-e_{N+1}\right)}\right)}{i s h\left(m_{N+1}-m_{\rho(i)}\right) \operatorname{sh}\left(e_{i}-e_{N+1}\right)} \\
& =\frac{1}{i^{N(N+1) / 2} \prod_{p<q}^{N+1} \operatorname{sh}\left(m_{p}-m_{q}\right) \prod_{i<j}^{N+1} \operatorname{sh}\left(e_{i}-e_{j}\right)} \sum_{\rho^{\prime} \in S^{N+1}}(-1)^{\rho^{\prime}} e^{2 \pi i \sum_{i=1}^{N} m_{\rho^{\prime}(i)}\left(e_{i}-e_{N+1}\right)}
\end{aligned}
$$

where in the last line we made use of the following identity ${ }^{4}$ :

$$
\sum_{\rho \in S^{N}}(-1)^{\rho} \prod_{i=1}^{N}\left(e^{m_{\rho(i)} \alpha_{i}}-e^{m_{N+1} \alpha_{i}}\right)=\sum_{\rho^{\prime} \in S^{N+1}}(-1)^{\rho^{\prime}} \prod_{i=1}^{N} e^{m_{\rho^{\prime}(i)} \alpha_{i}} .
$$

The last line in eq. (4.12) is precisely $\hat{\mathcal{Z}}^{T(S U(N+1)}$, this concludes our proof.

\subsection{Two maximal and one minimal puncture}

In this section we will study the theory on a sphere with two maximal and one minimal puncture. This theory is ugly in the Gaiotto-Witten sense and corresponds to $N^{2}$ free hypers. We will evaluate the partition function of the mirror theory the star shaped quiver obtained by gluing two full punctures $\mathcal{Z}_{N}^{\odot}$ and one abelian integral $\hat{\mathcal{Z}}_{N}^{U(1)}$, corresponding to a simple puncture $\mathcal{Z}^{\times}$, see Fig. 7 .

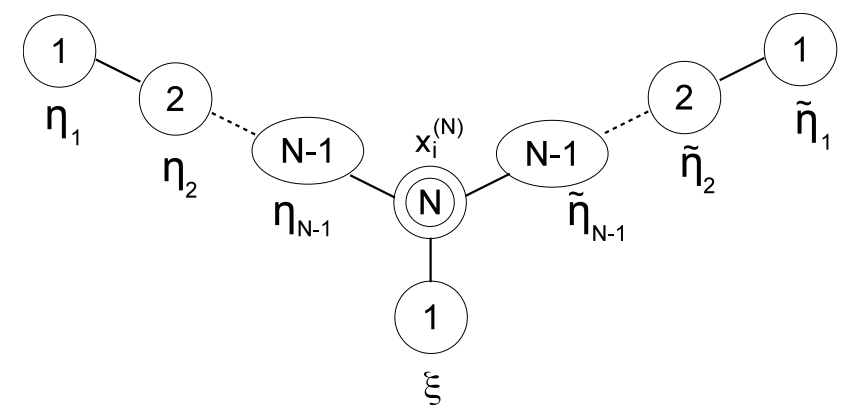

Figure 7. Two maximal and one minimal punctures

\footnotetext{
${ }^{4}$ The validity of this formula can be seen as follows. Separate all the $N ! \cdot 2^{N}$ terms on the L.H.S. according to the number of times they contain the factor $e^{m_{N+1}}$. If $K>1$, all the terms containing $m_{N+1}$ precisely $K$ times cancel out among themselves when performing the sum over the $S^{N}$-permutations $\rho$, due to the $(-1)^{\rho}$ prefactor. So we are left with $N$ ! terms that do not contain $m_{N+1}$ and $N$ ! $\cdot N$ terms that contain $m_{N+1}$ exactly once, which is precisely the content of the R.H.S.
} 
The partition function reads:

$$
\mathcal{Z}_{N}^{\times \odot \odot}(\xi, \tilde{e}, e)=\int \frac{\mathrm{d} a \mathrm{~d}^{N} x \delta\left(\sum x\right) \prod_{m<n} s h^{2}\left(x_{m}-x_{n}\right)}{N !} \frac{e^{2 \pi i \xi a}}{\prod_{i}^{N} \operatorname{ch}\left(x_{i}-a\right)} \mathcal{Z}_{N}^{\odot}\left(x ; e_{i}\right) \mathcal{Z}_{N}^{\odot}\left(x ; \tilde{e}_{i}\right)
$$

by plugging in the result for $\mathcal{Z}^{T(S U(N))}$ we obtain:

$$
\begin{gathered}
\frac{1}{i^{N(N-1)} \prod_{i<j}^{N} \operatorname{sh}\left(e_{i}-e_{j}\right) \prod_{i<j}^{N} \operatorname{sh}\left(\tilde{e}_{i}-\tilde{e}_{j}\right)} \sum_{\rho, \tilde{\rho}}(-1)^{\rho+\tilde{\rho}} \int \frac{\mathrm{d} a \mathrm{~d}^{N} x \delta\left(\sum x\right) e^{2 \pi i \xi a} e^{2 \pi i \sum_{j} x_{j}\left(e_{\rho(j)}+\tilde{e}_{\tilde{\rho}(j)}\right)}}{N ! \prod_{i}^{N} \operatorname{ch}\left(x_{i}-a\right)} \\
=\frac{1}{i^{N(N-1)} \prod_{i<j}^{N} \operatorname{sh}\left(e_{i}-e_{j}\right) \prod_{i<j}^{N} \operatorname{sh}\left(\tilde{e}_{i}-\tilde{e}_{j}\right)} \sum_{\rho}(-1)^{\rho} \int \frac{\mathrm{d} a \mathrm{~d}^{N} x \delta\left(\sum x\right) e^{2 \pi i \xi a} e^{2 \pi i \sum_{j} x_{j}\left(e_{\rho(j)}+\tilde{e}_{j}\right)}}{\prod_{i}^{N} \operatorname{ch}\left(x_{i}-a\right)},
\end{gathered}
$$

where we changed integration variable to remove one sum over permutations. We need the following integral with $A=\left(A_{1}, A_{2}, \ldots, A_{N}\right)=\left(\left\{e_{\rho(j)}+\tilde{e}_{j}\right\}\right)$ :

$$
\begin{aligned}
& \int \mathrm{d} a \mathrm{~d}^{N} x \delta\left(\sum x\right) \frac{e^{2 \pi i\left(\xi a+\sum_{j} x_{j} A_{j}\right)}}{\prod_{i}^{N} \operatorname{ch}\left(x_{i}-a\right)}=\int \mathrm{d} a \mathrm{~d}^{N} x \delta\left(\sum x+N a\right) \frac{e^{2 \pi i\left(\left(\xi+\sum_{i} A_{i}\right) a+\sum_{j} x_{j} A_{j}\right)}}{\prod_{i}^{N} \operatorname{ch}\left(x_{i}\right)}= \\
& =\frac{1}{N} \int \mathrm{d}^{N} x \frac{e^{2 \pi i \sum_{j} x_{j}\left(A_{j}-\left(\xi+\sum A_{i}\right) / N\right)}}{\prod_{i}^{N} \operatorname{ch}\left(x_{i}\right)}=\frac{1}{N \prod_{i}^{N} \operatorname{ch}\left(\xi / N-\left\langle A, h_{i}\right\rangle\right)},
\end{aligned}
$$

where $h_{i}$ are the weights of $S U(N)$ :

$$
\left(h_{j}\right)^{I}=\delta_{j}^{I}-\frac{1}{N} .
$$

The partition function becomes

$$
\begin{aligned}
\mathcal{Z}_{N}^{\times \odot \odot}(\xi, \tilde{\eta}, \eta)= & \frac{1}{i^{N(N-1)} \prod_{i<j}^{N} \operatorname{sh}\left(e_{i}-e_{j}\right) \prod_{i<j}^{N} \operatorname{sh}\left(\tilde{e}_{i}-\tilde{e}_{j}\right)} \times \\
& \times \sum_{\rho}(-1)^{\rho} \frac{1}{N \prod_{i}^{N} \operatorname{ch}\left(\xi / N-\left\langle e_{\rho(i)}, h_{i}\right\rangle-\left\langle\tilde{e}_{i}, h_{i}\right\rangle\right)} .
\end{aligned}
$$

Finally we used the Cauchy determinant formula to get:

$$
\mathcal{Z}_{N}^{\times \odot \odot}(\xi, \tilde{\eta}, \eta)=\frac{1}{i^{N(N-1)} N} \prod_{i, j}^{N} \frac{1}{\operatorname{ch}\left(\xi / N-\left\langle e_{i}, h_{i}\right\rangle-\left\langle\tilde{e}_{j}, h_{j}\right\rangle\right)},
$$

which is, up to a prefactor, the partition function of $N^{2}$ free hypers, as expected from mirror symmetry. 


\subsection{Two maximal and $k+2$ minimal}

In this section we study an infinite family of mirror theories associated to the sphere with two maximal and $k$ minimal punctures. On one side we have the linear quiver theories $S U(N)^{k+1}$ with $N+N$ fundamentals $m_{i}, \tilde{m}_{i}, i=1, \cdots N$ acted by a $U(N)^{2}$ flavour symmetry, and $k$ bi-fundamentals $M_{j}, j=1, \cdots k$ depicted in Fig. 8.

On the other side we have the the star shaped quivers $Z^{\times \cdots \times \odot \odot}$, obtained by gluing two full punctures $\mathcal{Z}^{\odot}$ and $k+2$ abelian integrals $\hat{\mathcal{Z}}_{N}^{U(1)}$ with FI's $\eta_{b}, \eta_{c}, \xi_{1} \cdots \xi_{k}$ and Cartan's $b, c, a_{1} \cdots a_{k}$ as indicated in Fig. 9.

We start from the linear quiver theory.

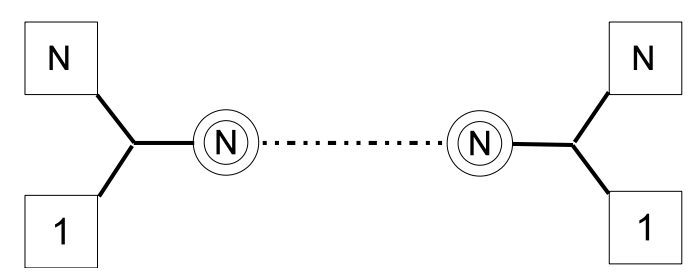

Figure 8. $S U(N)^{K}$ quiver.

The partition function reads:

$$
\begin{aligned}
\mathcal{Z}_{2 N}^{S U(N)^{k+1}} & =\frac{1}{(N !)^{k+1}} \int \prod_{i}^{N} \mathrm{~d} x_{i} \mathrm{~d} z_{i} \prod_{r}^{k-1} \mathrm{~d} y_{i}^{(r)} \delta\left(\sum_{i} x_{i}\right) \delta\left(\sum_{i} z_{i}\right) \delta\left(\sum_{i} y_{i}^{r}\right) \\
& \times \frac{\prod_{i<j}^{N} \operatorname{sh}\left(x_{i}-x_{j}\right)^{2} \prod_{r}^{k-1} \operatorname{sh}\left(y_{i}^{(r)}-y_{j}^{(r)}\right)^{2} \operatorname{sh}\left(z_{i}-z_{j}\right)^{2}}{\operatorname{ch}\left(x_{i}-m_{j}\right) \operatorname{ch}\left(y_{i}^{(1)}-x_{j}-M_{1}\right) \operatorname{ch}\left(y_{i}^{(2)}-y_{j}^{(1)}-M_{2}\right) \cdots \operatorname{ch}\left(z_{i}-y_{j}^{(k-1)}-M_{k}\right) \operatorname{ch}\left(z_{i}-\tilde{m}_{j}\right)} .
\end{aligned}
$$

We now use the Cauchy determinant $k+1$ times and get $k+2$ sums over permutations of $S_{N}$. By changing variables it is possible to reorder $k+1$ permutations and we find:

$$
\begin{aligned}
& \frac{1}{\prod_{i<j}^{N} \operatorname{sh}\left(m_{i}-m_{j}\right) \operatorname{sh}\left(\tilde{m}_{i}-\tilde{m}_{j}\right)} \sum_{\rho \in S^{N}}(-)^{\rho} \int \prod_{i}^{N} \mathrm{~d} x_{i} \mathrm{~d} z_{i} \prod_{r}^{k-1} \mathrm{~d} y_{i}^{(r)} \delta\left(\sum_{i} x_{i}\right) \delta\left(\sum_{i} z_{i}\right) \delta\left(\sum_{i} y_{i}^{r}\right) \\
& \frac{1}{\operatorname{ch}\left(x_{i}-m_{\rho(i)}\right) \operatorname{ch}\left(y_{i}^{(1)}-x_{i}-M_{1}\right) \operatorname{ch}\left(y_{i}^{(2)}-y_{i}^{(1)}-M_{2}\right) \cdots \operatorname{ch}\left(z_{i}-y_{i}^{(k-1)}-M_{k}\right) \operatorname{ch}\left(z_{i}-\tilde{m}_{i}\right)} .
\end{aligned}
$$

Now we shift $x_{i} \rightarrow x_{i}+m_{\rho(i)}, z_{i} \rightarrow z_{i}+\tilde{m}_{i}, y^{(r)} \rightarrow \sum_{j}^{r} y_{i}^{(j)}+x_{i}+m_{\rho(i)}+\sum_{j}^{r} M_{j}$ and rewrite the partition function as: 


$$
\begin{aligned}
\mathcal{Z}_{2 N}^{S U(N)^{k+1}}= & \frac{1}{\prod_{i<j}^{N} \operatorname{sh}\left(m_{i}-m_{j}\right) \operatorname{sh}\left(\tilde{m}_{i}-\tilde{m}_{j}\right)} \sum_{\rho \in S^{N}}(-)^{\rho} \int \prod_{i}^{N-1} \mathrm{~d} x_{i} \mathrm{~d} z_{i} \prod_{r}^{k-1} \mathrm{~d} y_{i}^{(r)} \\
& \times \frac{1}{\prod_{i}^{N-1} \operatorname{ch}\left(x_{i}\right) \operatorname{ch}\left(z_{i}\right) \operatorname{ch}\left(\sum_{i}^{N-1} x_{i}+\sum_{i}^{N} m_{i}\right) \operatorname{ch}\left(\sum_{i}^{N-1} z_{i}+\sum_{i}^{N} \tilde{m}_{i}\right)} \\
& \times \frac{1}{\prod_{r}^{k-1} \prod_{i}^{N-1} \operatorname{ch}\left(y_{i}^{(r)}\right) \operatorname{ch}\left(\sum_{i}^{N-1} y_{i}^{(r)}+N M_{r}\right)} \\
& \times \frac{1}{\prod_{i}^{N-1} \operatorname{ch}\left(z_{i}+\tilde{m}_{i}-\sum_{j}^{k} y_{i}^{(j)}-x_{i}-m_{\rho(i)}-\sum_{r}^{k} M_{r}\right)} \\
\times & \frac{1}{\operatorname{ch}\left(-\sum_{i}^{N-1}\left(z_{i}+\tilde{m}_{i}\right)+\sum_{i}^{N-1} \sum_{j}^{k} y_{i}^{(j)}+\sum_{i}^{N-1}\left(x_{i}+m_{\rho(i)}\right)+(N-1) \sum_{r}^{k-1} M_{r}-M_{k}\right)}
\end{aligned}
$$

Let's now look at the mirror star-shaped quiver.

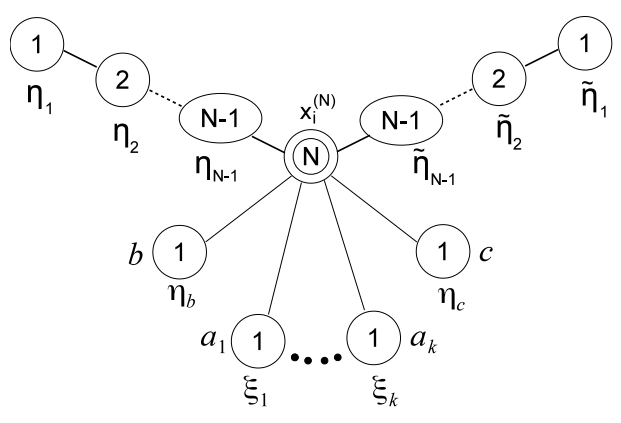

Figure 9. Mirror of two maximal and $k+2$ minimal punctures.

The partition function reads:

$$
\begin{aligned}
\mathcal{Z}_{N}^{\times \cdots \times \odot \odot}= & \int \frac{\mathrm{d}^{N} x \delta\left(\sum x\right) \prod_{i<j} \operatorname{sh}^{2}\left(x_{i}-x_{j}\right)}{N !} \mathcal{Z}_{N}^{\odot}\left(x ; e_{i}\right) \mathcal{Z}_{N}^{\odot}\left(x ; \tilde{e}_{i}\right) \\
& \times \int \mathrm{d} b \mathrm{~d} c \prod_{j}^{k} \mathrm{~d} a_{j} \frac{e^{2 \pi i\left(\eta_{b} b+\eta_{c} c\right)}}{\prod_{i}^{N} \operatorname{ch}\left(x_{i}-b\right) \operatorname{ch}\left(x_{i}-c\right)} \frac{e^{2 \pi i \sum_{j}^{k} \xi_{j} a_{j}}}{\prod_{i}^{N} \prod_{j}^{k} \operatorname{ch}\left(x_{i}-a_{j}\right)}
\end{aligned}
$$

Plugging in the exact expression for $\mathcal{Z}^{T(S U(N)}$ we find:

$$
\begin{aligned}
& \frac{1}{i^{N(N-1)} \prod_{i<j}^{N} \operatorname{sh}\left(e_{i}-e_{j}\right) \prod_{i<j}^{N} \operatorname{sh}\left(\tilde{e}_{i}-\tilde{e}_{j}\right)} \sum_{\rho}(-1)^{\rho} \int d^{N} x \delta\left(\sum x\right) e^{2 \pi i \sum_{j} x_{j}\left(e_{\rho(j)}+\tilde{e}_{j}\right)} \\
& \times \int \mathrm{d} b \mathrm{~d} c \prod_{j}^{k} \mathrm{~d} a_{j} \frac{e^{2 \pi i\left(\eta_{b} b+\eta_{c} c\right)}}{\prod_{i}^{N} \operatorname{ch}\left(x_{i}-b\right) \operatorname{ch}\left(x_{i}-c\right)} \frac{e^{2 \pi i \sum_{j}^{k} \xi_{j} a_{j}}}{\prod_{i}^{N} \prod_{j}^{k} \operatorname{ch}\left(x_{i}-a_{j}\right)}
\end{aligned}
$$


where we performed a change of integration variables to remove one sum over permutations.

We need the following integral:

$$
\begin{aligned}
& \int \mathrm{d}^{N} x \delta\left(\sum x\right) \mathrm{d} b \mathrm{~d} c \prod_{j}^{k} \mathrm{~d} a_{j} e^{2 \pi i \sum_{i}^{N} x_{i} A_{i}} \frac{e^{2 \pi i\left(\eta_{b} b+\eta_{c} c\right)}}{\prod_{i}^{N} \operatorname{ch}\left(x_{i}-b\right) \operatorname{ch}\left(x_{i}-c\right)} \frac{e^{2 \pi i \sum_{j}^{k} \xi_{j} a_{j}}}{\prod_{i}^{N} \prod_{j}^{k} \operatorname{ch}\left(x_{i}-a_{j}\right)}= \\
& =\int \mathrm{d}^{N} x \delta\left(\sum x\right) \mathrm{d} b \mathrm{~d} c \prod_{j}^{k} \mathrm{~d} a_{j} e^{2 \pi i \sum_{i}^{N} x_{i} A_{i}} e^{2 \pi i\left(\eta_{b} b+\eta_{c} c\right)} e^{2 \pi i \sum_{j}^{k} \xi_{j} a_{j}} \\
& \times \prod_{i}^{N} \int \mathrm{d} s_{i}^{(1)} \mathrm{d} s_{i}^{(2)} \frac{e^{2 \pi i\left(s_{i}^{(1)}\left(x_{i}-b\right)+s_{i}^{(2)}\left(x_{i}-c\right)\right)}}{c h s_{i}^{(1)} c h s_{i}^{(2)}} \prod_{i}^{N} \prod_{j}^{k} \int \mathrm{d} t_{i}^{(j)} \frac{e^{2 \pi i t_{i}^{(j)}\left(x_{i}-a_{j}\right)}}{c h t_{i}^{(j)}}
\end{aligned}
$$

with $A_{i}=e_{\rho(i)}+\tilde{e}_{i}$. The integration over $b, c, a_{j}, x_{i}$ produces the following deltas:

$$
\begin{array}{ll}
\delta\left(-\sum_{i}^{N} s_{i}^{(1)}+\eta_{b}\right), & \delta\left(-\sum_{i}^{N} s_{i}^{(2)}+\eta_{c}\right), \\
\delta\left(-\sum_{i}^{N} t_{i}^{(j)}+\xi_{j}\right), \quad j=1, \cdots k & \\
\delta\left(A_{i}-A_{N}+s_{i}^{(1)}-s_{N}^{(1)}+s_{i}^{(2)}-s_{N}^{(2)}+\sum_{j}^{k} t_{i}^{(j)}-\sum_{j}^{k} t_{N}^{(j)}\right), \quad i=1, \cdots N-1
\end{array}
$$

we choose as independent variables $s_{i}^{(1)}, s_{i}^{(2)}$ and $t_{i}^{(j)}, j=1, \cdots k-1$ with $i=1, \cdots N-1$. From the first three deltas we obtain:

$$
s_{N}^{(1)}=-\sum_{i}^{N-1} s_{i}^{(1)}+\eta_{b}, \quad s_{N}^{(2)}=-\sum_{i}^{N-1} s_{i}^{(2)}+\eta_{c}, \quad t_{N}^{(j)}=-\sum_{i}^{N-1} t_{i}^{(j)}+\xi_{j}, \quad j=1, \cdots k .
$$

By manipulating the system we obtain the following equation:

$$
N A_{i}-\sum_{l}^{N} A_{l}+N s_{i}^{(1)}-\eta_{b}+N s_{i}^{(2)}-\eta_{c}+\sum_{j}^{k-1}\left(N t_{i}^{(j)}-\xi_{j}\right)+N t_{i}^{(k)}-\xi_{k}=0
$$

from which we get:

$$
-t_{i}^{(k)}=\frac{-\eta_{b}-\eta_{c}-\sum_{j}^{k} \xi_{j}+N A_{i}-\sum_{l}^{N} A_{l}}{N}+s_{i}^{(1)}+s_{i}^{(2)}+\sum_{j}^{k-1} t_{i}^{(j)}, \quad i=1, \cdots N-1,
$$


and

$$
\begin{aligned}
t_{N}^{(k)}= & -\sum^{N-1} t_{i}^{(k)}+\xi_{k}=\frac{(N-1)\left(-\eta_{b}-\eta_{c}-\sum_{l}^{N} A_{l}\right)-(N-1) \sum_{j}^{k} \xi_{j}+\xi_{k}+N \sum_{i}^{N-1} A_{i}}{N}+ \\
& +\sum_{i}^{N-1}\left(s_{i}^{(1)}+s_{i}^{(2)}+\sum_{j}^{k-1} t_{i}^{(j)}\right) .
\end{aligned}
$$

In terms of these variables the partition function can be rewritten as:

$$
\begin{aligned}
\mathcal{Z}_{N}^{\times \cdots \times \odot \odot}= & \frac{1}{i^{N(N-1)}} \frac{\mathcal{J}}{\prod_{i<j}^{N} \operatorname{sh}\left(e_{i}-e_{j}\right) \operatorname{sh}\left(\tilde{e}_{i}-\tilde{e}_{j}\right)} \sum_{\rho \in S^{N}}(-)^{\rho} \int \prod_{i}^{N-1} \mathrm{~d} s_{i}^{(1)} \mathrm{d} s_{i}^{(2)} \prod_{r}^{k-1} \mathrm{~d} y_{i}^{(r)} \\
& \times \frac{1}{\prod_{i}^{N-1} \operatorname{ch}\left(s_{i}^{(1)}\right) \operatorname{ch}\left(s_{i}^{(2)}\right) \operatorname{ch}\left(\sum_{i}^{N-1} s_{i}^{(1)}-\eta_{b}\right) \operatorname{ch}\left(\sum_{i}^{N-1} s_{i}^{(2)}-\eta_{c}\right)} \\
& \times \frac{1}{\prod_{j}^{k-1} \prod_{i}^{N-1} \operatorname{ch}\left(t_{i}^{(j)}\right) \operatorname{ch}\left(\sum_{i}^{N-1} t_{i}^{(j)}-\xi_{j}\right)} \\
& \times \frac{1}{\prod_{i}^{N-1} \operatorname{ch}\left(\frac{-\eta_{b}-\eta_{c}-\sum_{j}^{k} \xi_{j}+N A_{i}-\sum_{l}^{N} A_{l}}{N}+s_{i}^{(1)}+s_{i}^{(2)}+\sum_{j}^{k-1} t_{i}^{(j)}\right)} \\
\times & \frac{1}{\operatorname{ch}\left(\frac{(N-1)\left(-\eta_{b}-\eta_{c}-\sum_{l}^{N} A_{l}\right)+(N-1) \sum_{j}^{k} \xi_{j}-\xi_{k}+N \sum_{i}^{N-1} A_{i}}{N}+\sum_{i}^{N-1}\left(s_{i}^{(1)}+s_{i}^{(2)}+\sum_{j}^{k-1} t_{i}^{(j)}\right)\right)} .
\end{aligned}
$$

Where $\mathcal{J}$ is a constant coming from the Jacobian. It is easy to see that this expression

coincides with the partition function of the mirror theory eq. (4.20), when inserting the following dictionary:

$$
\begin{array}{rlrl}
\eta_{b}=\sum_{i}^{N} m_{i}, & \eta_{c}=-\sum_{i}^{N} \tilde{m}, & e_{i}=-m_{i}, \quad \tilde{e}_{i}=\tilde{m}_{i}, \\
\xi_{j}=N M_{j}, & j=1, \cdots k,
\end{array}
$$

notice that the two small tails with FI's $\eta_{b, c}$ carry the extra $U(1)$ flavour symmetry (with charge \pm 1 ) of the two full puncture. While the other $k$ small tails carry the $U(1)$ symmetry associated to the bi-fundamentals.

\section{$5 \quad T_{N}$ theories}

We now move to the study of generalised quiver theories. The natural building blocks to construct these theories are partition functions associated to spheres with 3 punctures. Our first goal will be the computation of the partition function of the $T_{N}$ theory 
$\mathcal{Z}_{T_{N}}\left(m_{i}, \tilde{m}_{j}, \hat{m}_{k}\right)$, associated to the sphere with 3 full punctures, which depends on three sets of $S U(N)$ masses. Another block that we will need is the partition function of the ugly theory associated to the sphere with two full and one minimal puncture $\mathcal{Z}\left(m_{i}, \tilde{m}_{j}, \eta\right)$, which we computed in section 4.2 . Finally we need the block $\mathcal{Z}\left(m_{i}, \eta_{a}, \eta_{b}\right)$ for the bad theory associated to the sphere with one maximal and two minimal punctures which is given by:

$$
\begin{aligned}
\mathcal{Z}\left(m_{i}, \eta_{a}, \eta_{b}\right)= & \frac{1}{i^{N(N-1) / 2} \prod_{i<j}^{N} \operatorname{sh}\left(m_{i}-m_{j}\right)} \int \mathrm{d}^{N} z_{i} \delta\left(\sum z_{i}\right) \prod_{i<j} \operatorname{sh}\left(z_{i}-z_{j}\right) e^{2 \pi i \sum_{j} z_{j} m_{j}} \\
& \times \int \mathrm{d} a \mathrm{~d} b \frac{e^{2 \pi i\left(\eta_{a} a+\eta_{b} b\right)}}{\prod_{i}^{N} \operatorname{ch}\left(z_{i}-a\right) \operatorname{ch}\left(z_{i}-b\right)} .
\end{aligned}
$$

In section 5.2 we will show how to obtain generalised quiver theories by gluing the $\mathcal{Z}\left(m_{i}, \eta_{a}, \eta_{b}\right), \mathcal{Z}\left(m_{i}, \tilde{m}_{j}, \eta\right)$ and $\mathcal{Z}_{T_{N}}\left(m_{i}, \tilde{m}_{j}, \hat{m}_{k}\right)$ blocks.

\subsection{The $T_{N}$ block}

The $T_{N}$ theory is not Lagrangian and in principle one can not use localisation of the path integral to evaluate the partition function, however, assuming mirror symmetry we can obtain the $T_{N}$ partition function from its mirror: the Lagrangian star shaped quiver theory obtained gluing three $Z_{N}^{\odot}$ blocks depicted in Fig. 10.

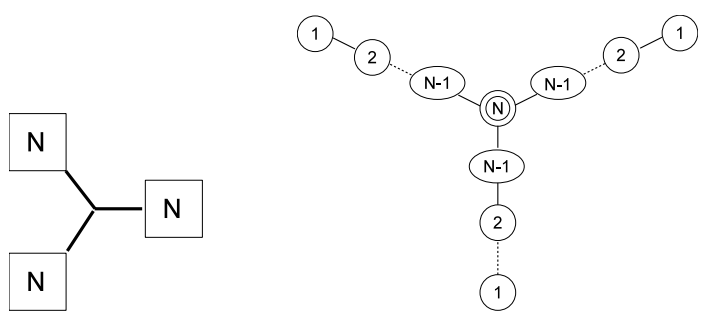

Figure 10. The $T_{N}$ theory and its mirror.

The partition function reads:

$$
\begin{aligned}
\mathcal{Z}_{N}^{\odot \odot \odot}\left(e_{i}, \tilde{e}_{j}, \hat{e}_{k}\right)= & \int \frac{\mathrm{d}^{N} x \delta\left(\sum x\right) \prod_{i<j} s^{2}\left(x_{i}-x_{j}\right)}{N !} \mathcal{Z}_{N}^{\odot}\left(x ; e_{i}\right) \mathcal{Z}_{N}^{\odot}\left(x ; \tilde{e}_{i}\right) \mathcal{Z}_{N}^{\odot}\left(x ; \hat{e}_{k}\right)= \\
= & \frac{1}{i^{3 N(N-1) / 2} \prod_{i<j}^{N} \operatorname{sh}\left(e_{i}-e_{j}\right) \operatorname{sh}\left(\tilde{e}_{i}-\tilde{e}_{j}\right) \operatorname{sh}\left(\hat{e}_{i}-\hat{e}_{j}\right)} \sum_{\rho, \tilde{\rho}, \hat{\rho}}(-1)^{\rho+\tilde{\rho}+\hat{\rho}} \\
& \times \int \frac{d^{N} x \delta\left(\sum x\right)}{N ! \prod_{i<j} \operatorname{sh}\left(x_{i}-x_{j}\right)} e^{2 \pi i \sum x_{i}\left(e_{\rho(i)}+\tilde{e}_{\tilde{\rho}(i)}+\hat{e}_{\hat{\rho}(i)}\right)} .
\end{aligned}
$$


Inserting the dictionary $e_{i} \rightarrow m_{i}, \tilde{e}_{i} \rightarrow \tilde{m}_{i}$ and $\hat{e}_{i} \rightarrow \hat{m}_{i}$ we obtain the partition function of the $T_{N}$ theory:

$$
\begin{aligned}
\hat{\mathcal{Z}}_{T_{N}}\left(m_{i}, \tilde{m}_{j}, \hat{m}_{k}\right)= & \frac{1}{i^{3 N(N-1) / 2} \prod_{i<j}^{N} \operatorname{sh}\left(m_{i}-m_{j}\right) \operatorname{sh}\left(\tilde{m}_{i}-\tilde{m}_{j}\right) \operatorname{sh}\left(\hat{m}_{i}-\hat{m}_{j}\right)} \\
& \times \sum_{\rho, \tilde{\rho}, \hat{\rho}}(-1)^{\rho+\tilde{\rho}+\hat{\rho}} \int \frac{\mathrm{d}^{N} x \delta\left(\sum x\right)}{N ! \prod_{i<j} \operatorname{sh}\left(x_{i}-x_{j}\right)} e^{2 \pi i \sum x_{i}\left(m_{\rho(i)}+\tilde{m}_{\tilde{\rho}(i)}+\hat{m}_{\hat{\rho}(i)}\right)} .
\end{aligned}
$$

This expression has a manifest $\left(S_{N}\right)^{3}$ symmetry. In particular, thanks to the symmetrisation, the partition function is finite. We will see how this works in detail for the $T_{3}$ case.

The $T_{3}$ theory Let's now focus on the $T_{3}$ case. We need to compute the following divergent integral:

$$
F\left(C_{i}\right)=\int \frac{\mathrm{d}^{3} x \delta\left(\sum x\right)}{\prod_{i<j} \operatorname{sh}\left(x_{i}-x_{j}\right)} e^{2 \pi i \sum C_{i} x_{i}}
$$

with $C=\left(C_{1}, C_{2}, C_{3}\right)=\left(\left\{e_{\rho(i)}+\tilde{e}_{\tilde{\rho}(i)}+\hat{e}_{\hat{\rho}(i)}\right\}\right)$. We shift $x_{1} \rightarrow x_{1}+x_{3}$ and $x_{2} \rightarrow x_{2}+x_{3}$ and get:

$$
\begin{aligned}
& \int \frac{\mathrm{d} x_{1} \mathrm{~d} x_{2} \mathrm{~d} x_{3} \delta\left(x_{1}+x_{2}+3 x_{3}\right)}{\operatorname{sh}\left(x_{1}-x_{2}\right) \operatorname{sh}\left(x_{1}\right) \operatorname{sh}\left(x_{2}\right)} e^{2 \pi i\left(C_{1} x_{1}+C_{2} x_{2}+\sum_{i}^{3} C_{i} x_{3}\right)}=\frac{1}{3} \int \frac{\mathrm{d} x_{1} \mathrm{~d} x_{2} e^{2 \pi i\left(B_{1} x_{1}+B_{2} x_{2}\right)}}{\operatorname{sh}\left(x_{1}-x_{2}\right) \operatorname{sh}\left(x_{1}\right) \operatorname{sh}\left(x_{2}\right)} \\
& =\frac{i^{3}}{24} \int \mathrm{d} a \mathrm{~d} b \mathrm{~d} c \int \mathrm{d} x_{1} \mathrm{~d} x_{2} e^{2 \pi i\left(B_{1} x_{1}+B_{2} x_{2}\right)} e^{2 \pi i\left(a\left(x_{1}-x_{2}\right)+b x_{1}+c x_{2}\right)} \operatorname{th}(a) \operatorname{th}(b) \operatorname{th}(c)= \\
& =\frac{i^{3}}{24} \int \operatorname{dath}(a) \operatorname{th}\left(a+B_{1}\right) \operatorname{th}\left(a-B_{2}\right),
\end{aligned}
$$

with $B_{i}=\left\langle C, h_{i}\right\rangle$. Where we used that:

$$
\frac{1}{\sinh (x)}=\frac{i}{2} \int \mathrm{d} a e^{-2 \pi i a x} \operatorname{th}(a)
$$

In order to compute the last integral we introduce a $F I$ parameter, playing the role of an IR regulator:

$$
\begin{aligned}
& \int \mathrm{d} a \operatorname{th}(a) \operatorname{th}\left(a+B_{1}\right) \operatorname{th}\left(a-B_{2}\right) e^{2 \pi i \xi a}= \\
& =\frac{i}{\operatorname{sh}(\xi)}\left(-\frac{\operatorname{ch} B_{1} \operatorname{ch}\left(B_{2}\right)}{\operatorname{sh}\left(B_{1}\right) \operatorname{sh}\left(B_{2}\right)}+\frac{e^{-2 \pi i \xi B_{1}} \operatorname{ch}\left(B_{1}\right) \operatorname{ch}\left(B_{1}+B_{2}\right)}{\operatorname{sh}\left(B_{1}\right) \operatorname{sh}\left(B_{1}+B_{2}\right)}+\frac{e^{2 \pi i \xi B_{2}} \operatorname{ch}\left(B_{2}\right) \operatorname{ch}\left(B_{1}+B_{2}\right)}{\operatorname{sh}\left(B_{1}+B_{2}\right) \operatorname{sh}\left(B_{2}\right)}\right)
\end{aligned}
$$


and expand the result for $\xi \rightarrow 0$

$$
=\frac{i}{2 \pi \xi}+\left(B_{1} \operatorname{coth} B_{1}-B_{2} \operatorname{coth} B_{2}\right) \operatorname{coth}\left(B_{1}+B_{2}\right)
$$

Notice that the divergent term cancels out thanks to the sum over the 3 sets of $S_{3}$ permutations. The on-shell ${ }^{5}$ partition function reads:

$$
\begin{aligned}
\mathcal{Z}_{T_{3}}\left(m_{i}, \tilde{m}_{j}, \hat{m}_{k}\right)=\frac{1}{3 ! \prod_{i<j}^{3} \operatorname{sh}\left(m_{i}-m_{j}\right) \operatorname{sh}\left(\tilde{m}_{i}-\tilde{m}_{j}\right) \operatorname{sh}\left(\hat{m}_{i}-\hat{m}_{j}\right)} \sum_{\rho, \tilde{\rho}, \hat{\rho}}(-1)^{\rho+\tilde{\rho}+\hat{\rho}} \\
\quad \times\left(m_{\rho(1)}+\tilde{m}_{\tilde{\rho}(1)}+\hat{m}_{\hat{\rho}(1)}\right) \operatorname{coth}\left(m_{\rho(1)}+\tilde{m}_{\tilde{\rho}(1)}+\hat{m}_{\hat{\rho}(1)}\right) \operatorname{coth}\left(m_{\rho(3)}+\tilde{m}_{\tilde{\rho}(3)}+\hat{m}_{\hat{\rho}(3)}\right) .
\end{aligned}
$$

\subsection{Consistency checks from S-duality invariance}

We will now glue our building blocks to obtain generalised quiver theories associated to spheres with arbitrary punctures. The partition functions we will construct must satisfy an important consistency condition: they must be independent on the particular pants-decomposition we choose to perform the gluing. This is a consequence of the fact that our theories are independent on the complex structure of the punctured sphere and they have the structure of a 2d TQFT. This has been recently pointed out in [19]. The super-conformal index of a $4 d$ theory on a punctured Riemann surface, which is computed by a $2 \mathrm{~d}$ TQFT $[20,21]$, has been shown to reduce, in a certain limit, to the $3 \mathrm{~d}$ partition function associated to the same punctured Riemann surface [19, 22]. It is then expected that the $3 \mathrm{~d}$ partition function will inherit the TQFT structure from the index.

To test our blocks, we will show that they satisfy the operator algebra of a $2 \mathrm{~d}$ TQFT, in particular we will prove the associativity relation indicated in Fig. 11, stating that the partition function of the sphere with four full punctures can be obtained equivalently as:

$$
\begin{aligned}
\mathcal{Z}\left(m_{i}^{(1)}, m_{j}^{(2)}, m_{k}^{(3)}, m_{l}^{(4)}\right) & =" \sum_{y_{n}} " \mathcal{Z}_{T_{N}}\left(m_{i}^{(1)}, m_{j}^{(2)}, y_{n}\right) \mathcal{Z}_{T_{N}}\left(y_{n}, m_{k}^{(3)}, m_{l}^{(4)}\right)= \\
& =" \sum_{y_{n}} " \mathcal{Z}_{T_{N}}\left(m_{i}^{(1)}, m_{j}^{(4)}, y_{n}\right) \mathcal{Z}_{T_{N}}\left(y_{n}, m_{k}^{(3)}, m_{l}^{(2)}\right)=\mathcal{Z}\left(m_{i}^{(1)}, m_{j}^{(4)}, m_{k}^{(3)}, m_{l}^{(2)}\right)
\end{aligned}
$$

\footnotetext{
${ }^{5}$ On-shell $\sum_{i} m_{i}=0$ and $\left\langle m, h_{i}\right\rangle=m_{i}$.
} 


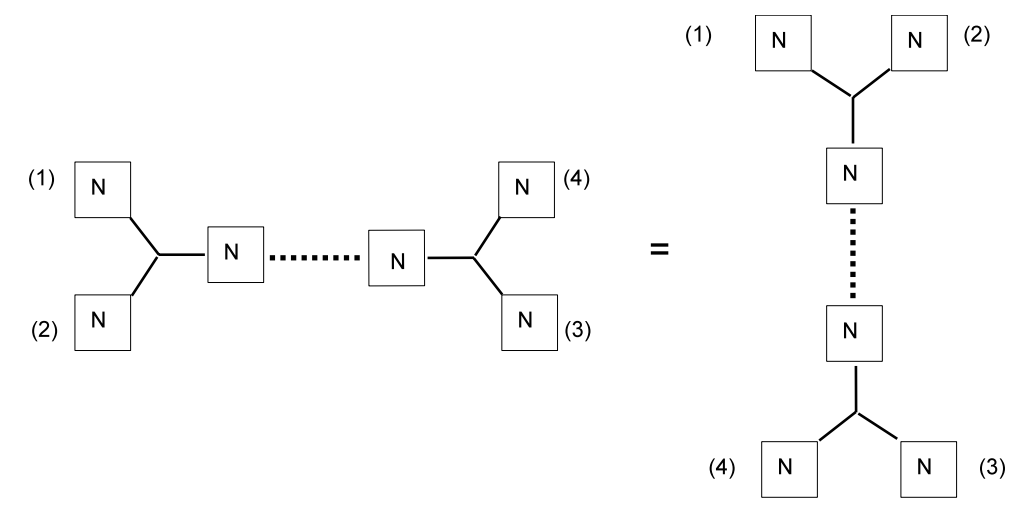

Figure 11. S-duality invariance as associativity relation for $T_{N}$ blocks.

To glue two $T_{N}$ blocks we simply gauge one of the $S U(N)$ flavour symmetry and we get:

$$
\begin{aligned}
& \mathcal{Z}\left(m_{i}^{(1)}, m_{j}^{(2)}, m_{k}^{(3)}, m_{l}^{(4)}\right)=\int \frac{\mathrm{d}^{N} y_{i} \delta\left(\sum y_{i}\right) \prod_{i<j} s h^{2}\left(y_{i}-y_{j}\right)}{N !} \mathcal{Z}_{T_{N}}\left(m_{i}^{(1)}, m_{j}^{(2)}, y_{n}\right) \mathcal{Z}_{T_{N}}\left(y_{n}, m_{k}^{(3)}, m_{l}^{(4)}\right)= \\
& =\frac{\sum_{\rho^{(1)}, \rho^{(2)}, \rho^{(3)}, \rho^{(4)}}(-1)^{\rho^{(1)}+\rho^{(2)}+\rho^{(3)}+\rho^{(4)}}}{\prod_{i<j}^{N} \operatorname{sh}\left(m_{i}^{(1)}-m_{j}^{(1)}\right) \operatorname{sh}\left(m_{i}^{(2)}-m_{j}^{(2)}\right) \operatorname{sh}\left(m_{i}^{(3)}-m_{j}^{(3)}\right) \operatorname{sh}\left(m_{i}^{(4)}-m_{j}^{(4)}\right)} \\
& \times \int \frac{\mathrm{d}^{N} y_{i} \delta\left(\sum y_{i}\right)}{N !} \int \frac{\mathrm{d}^{N} x \delta\left(\sum x\right)}{\prod_{i<j} \operatorname{sh}\left(x_{i}-x_{j}\right)} e^{2 \pi i \sum x_{i}\left(m_{\rho(1)(i)}^{(1)}+m_{\rho(2)(i)}^{(2)}+y_{i}\right)} \\
& \times \int \frac{\mathrm{d}^{N} z \delta\left(\sum z\right)}{\prod_{i<j} \operatorname{sh}\left(z_{i}-z_{j}\right)} e^{2 \pi i \sum z_{i}\left(m_{\rho(3)(i)}^{(3)}+m_{\rho(4)}^{(4)}+y_{i}\right)} .
\end{aligned}
$$

The integration over $y_{i}$ produces a delta function setting $z_{i}=x_{i}$ and we obtain:

$$
\begin{aligned}
& \frac{1}{\prod_{i<j}^{N} \operatorname{sh}\left(m_{i}^{(1)}-m_{j}^{(1)}\right) \operatorname{sh}\left(m_{i}^{(2)}-m_{j}^{(2)}\right) \operatorname{sh}\left(m_{i}^{(3)}-m_{j}^{(3)}\right) \operatorname{sh}\left(m_{i}^{(4)}-m_{j}^{(4)}\right)} \\
& \quad \sum_{\rho^{(1)}, \rho^{(2)}, \rho^{(3)}, \rho^{(4)}}(-1)^{\rho^{(1)}+\rho^{(2)}+\rho^{(3)}+\rho^{(4)}} \times \int \frac{\mathrm{d}^{N} x_{i} \delta\left(\sum x_{i}\right)}{N !} \frac{e^{2 \pi i \sum x_{i}\left(m_{\rho^{(1)}(i)}^{(1)}+m_{\rho^{(2)}(i)}^{(2)}+m_{\rho^{(3)}(i)}^{(3)}+m_{\rho^{(4)}(i)}^{(4)}\right)}}{\prod_{i<j} \operatorname{sh}\left(x_{i}-x_{j}\right)^{2}} .
\end{aligned}
$$

This expression is manifestly invariant under permutations of the $m^{(I)}$ 's and thus the associativity property eq. (5.10) is satisfied.

As a further test we show that $S U(N)$ theory with $N_{f}=2 N$ can be obtained in two ways. The first way, depicted on the left in Fig. 12, corresponds to gluing a $T_{N}$ 
block $\mathcal{Z}_{T_{N}}\left(m_{i}, \tilde{m}_{j}, y_{k}\right)$ and a bad block $\mathcal{Z}\left(y_{k}, \eta_{a}, \eta_{b}\right)$ with $\eta_{a}=\sum_{i} m_{i}, \eta_{b}=\sum_{i} \tilde{m}_{i}$. The second way, depicted on the right in Fig. 12, corresponds to gluing two ugly blocks $\mathcal{Z}\left(m_{i}, \eta_{a}, y_{k}\right)$ and $\mathcal{Z}\left(y_{k}, \eta_{b}, \tilde{m}_{j}\right)$.

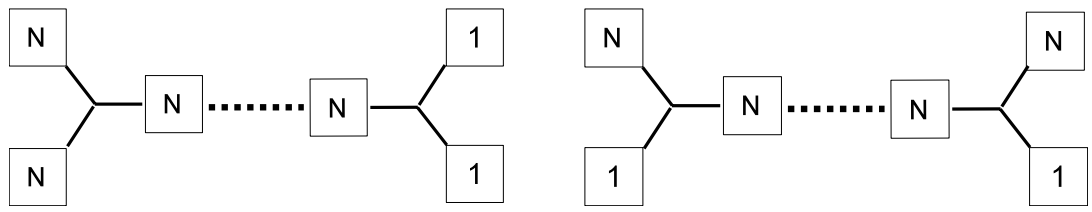

Figure 12. Two equivalent gluing for the $S U(N)$ theory with $N_{f}=2 N$,

Let's start with the first gluing:

$$
\begin{aligned}
& \mathcal{Z}_{2 N}^{S U(N)}\left(m_{i}, \tilde{m}_{j}\right)=\int \frac{\mathrm{d}^{N} y_{i} \delta\left(\sum y_{i}\right) \prod_{i<j} s h^{2}\left(y_{i}-y_{j}\right)}{N !} \mathcal{Z}_{T_{N}}\left(m_{i}, \tilde{m}_{j}, y_{k}\right) \mathcal{Z}\left(y_{k}, \eta_{a}, \eta_{b}\right)= \\
& =\frac{1}{i^{N(N-1)} \prod_{i<j}^{N} \operatorname{sh}\left(m_{i}-m_{j}\right) \operatorname{sh}\left(\tilde{m}_{i}-\tilde{m}_{j}\right)} \sum_{\rho, \rho^{\prime}}(-1)^{\rho+\rho^{\prime}} \int \frac{\mathrm{d}^{N} y_{i} \delta\left(\sum y_{i}\right)}{N !} \\
& \times \int \frac{\mathrm{d}^{N} x \delta\left(\sum x\right)}{\prod_{i<j} \operatorname{sh}\left(x_{i}-x_{j}\right)} e^{2 \pi i \sum x_{i}\left(m_{\rho(i)}+\tilde{m}_{\rho^{\prime}(i)}+y_{i}\right)} \int \frac{\mathrm{d} a \mathrm{~d} b \mathrm{~d}^{N} z_{i} \delta\left(\sum z_{i}\right) e^{2 \pi i\left(\eta_{a} a+\eta_{b} b\right)} e^{2 \pi i \sum_{j} z_{j} y_{j}} \prod_{i<j} \operatorname{sh}\left(z_{i}-z_{j}\right)}{\prod_{i}^{N} \operatorname{ch}\left(z_{i}-a\right) \operatorname{ch}\left(z_{i}-b\right)},
\end{aligned}
$$

the integration over $y_{i}$ sets $x_{i}=z_{i}$ and we obtain:

$\frac{1}{i^{N(N-1)} \prod_{i<j}^{N} \operatorname{sh}\left(m_{i}-m_{j}\right) \operatorname{sh}\left(\tilde{m}_{i}-\tilde{m}_{j}\right)} \sum_{\rho, \rho^{\prime}}(-1)^{\rho+\rho^{\prime}} \int \frac{\mathrm{d}^{N} x_{i} \delta\left(\sum x_{i}\right)}{N !} \frac{e^{2 \pi i \sum x_{i}\left(e_{\rho(i)}+\tilde{e}_{\rho^{\prime}(i)}\right)} e^{2 \pi i\left(\eta_{a} a+\eta_{b} b\right)}}{\prod_{i}^{N} \operatorname{ch}\left(z_{i}-a\right) \operatorname{ch}\left(z_{i}-b\right)}$.

For the second gluing we get:

$$
\begin{aligned}
& \mathcal{Z}_{2 N}^{S U(N)}\left(m_{i}, \tilde{m}_{j}\right)=\int \frac{\mathrm{d}^{N} y_{i} \delta\left(\sum y_{i}\right) \prod_{i<j} s h^{2}\left(y_{i}-y_{j}\right)}{N !} \mathcal{Z}\left(m_{i}, \eta_{a}, y_{k}\right) \mathcal{Z}\left(y_{k}, \eta_{b}, \tilde{m}_{j}\right)= \\
= & \frac{1}{i^{N(N-1)} \prod_{i<j}^{N} \operatorname{sh}\left(m_{i}-m_{j}\right) \operatorname{sh}\left(\tilde{m}_{i}-\tilde{m}_{j}\right)} \sum_{\rho, \rho^{\prime}}(-1)^{\rho+\rho^{\prime}} \int \frac{\mathrm{d}^{N} y_{i} \delta\left(\sum y_{i}\right)}{N !} \\
& \times \int \mathrm{d}^{N} x \delta\left(\sum x\right) e^{2 \pi i \sum x_{i}\left(m_{\rho(i)}+y_{i}\right)} \frac{e^{2 \pi i \eta_{a} a}}{c h\left(x_{i}-a\right)} \int \mathrm{d}^{N} z \delta\left(\sum z\right) e^{2 \pi i \sum z_{i}\left(\tilde{m}_{\rho^{\prime}(i)}+y_{i}\right)} \frac{e^{2 \pi i \eta_{b} b}}{\operatorname{ch}\left(z_{i}-b\right)},
\end{aligned}
$$

integrating over $y_{i}$ we obtain a delta leading to $x_{i}=z_{i}$ leading again to the result in eq. $(5.14)$. 


\section{Conclusions}

In this paper we developed a complete formalism to compute partition functions of generalised three-dimensional quiver theories deformed by mass and FI parameters. We used the mirror description in terms of Lagrangian star shaped quivers combined with localisation techniques.

One of our main results is the explicit evaluation of the partition function of the $T(S U(N))$ quiver theory as a function of the FI and mass parameters. The $T(S U(N))$ tail, mirror of the full puncture, is the fundamental building block to evaluate the partition function of generic star shaped quiver theories.

We provided several non-perturbative checks of the mirror realisation in terms of star shaped quivers [5] by showing that partition functions of mirror pairs of Lagrangian theories, are equal provided we exchange masses and FI's.

We then assumed mirror symmetry to find the partition function of non-Lagrangian theories in terms of the star-shaped mirrors. In particular we computed the partition function of the $T_{N}$ theory giving an explicit result for the $T_{3}$ case.

In this paper we only consider full punctures or minimal punctures. It is however very simple to extend our results to the case where punctures specified by generic Young tableaux with $\mathrm{N}$ boxes.

An interesting extension of our work would be to evaluate expectations values of supersymmetric observables such as Wilson Loops in the $3 \mathrm{~d}$ generalised quiver theories. With our explicit results for partition functions it should be possible to determine the mirror dual of these observables.

\section{Acknowledgments}

S.P would like to thank G. Bonelli, A. Brini and F. Passerini for useful comments on the draft. The work of S.P. is supported by a Marie Curie Intra-European Fellowship: FP7-PEOPLE-2009-IEF.

\section{References}

[1] D. Gaiotto, "N=2 dualities," [arXiv:0904.2715 [hep-th]].

[2] P. C. Argyres and N. Seiberg, "S-duality in N=2 supersymmetric gauge theories," JHEP 0712 (2007) 088 [arXiv:0711.0054 [hep-th]].

[3] J. A. Minahan and D. Nemeschansky, "An N=2 superconformal fixed point with $\mathrm{E}(6)$ global symmetry," Nucl. Phys. B 482 (1996) 142 [arXiv:hep-th/9608047]. 
[4] K. A. Intriligator, N. Seiberg, "Mirror symmetry in three-dimensional gauge theories," Phys. Lett. B387, 513-519 (1996). [hep-th/9607207].

[5] F. Benini, Y. Tachikawa, D. Xie, "Mirrors of 3d Sicilian theories," JHEP 1009, 063 (2010). [arXiv:1007.0992 [hep-th]].

[6] A. Hanany, E. Witten, "Type IIB superstrings, BPS monopoles, and three-dimensional gauge dynamics," Nucl. Phys. B492, 152-190 (1997). [hep-th/9611230].

[7] V. Pestun, "Localization of gauge theory on a four-sphere and supersymmetric Wilson loops," arXiv:0712.2824 [hep-th].

[8] A. Kapustin, B. Willett, I. Yaakov, "Exact Results for Wilson Loops in Superconformal Chern-Simons Theories with Matter," JHEP 1003, 089 (2010). [arXiv:0909.4559 [hep-th]].

[9] N. Drukker, M. Marino, P. Putrov, "From weak to strong coupling in ABJM theory," [arXiv:1007.3837 [hep-th]].

[10] R. C. Santamaria, M. Marino and P. Putrov, "Unquenched flavor and tropical geometry in strongly coupled Chern-Simons-matter theories," arXiv:1011.6281 [hep-th].

[11] C. P. Herzog, I. R. Klebanov, S. S. Pufu, T. Tesileanu, "Multi-Matrix Models and Tri-Sasaki Einstein Spaces," Phys. Rev. D83, 046001 (2011). [arXiv:1011.5487 [hep-th]].

[12] M. Marino, "Lectures on localization and matrix models in supersymmetric Chern-Simons-matter theories," [arXiv:1104.0783 [hep-th]].

[13] D. L. Jafferis, "The Exact Superconformal R-Symmetry Extremizes Z," [arXiv:1012.3210 [hep-th]].

[14] N. Hama, K. Hosomichi, S. Lee, "Notes on SUSY Gauge Theories on Three-Sphere," JHEP 1103, 127 (2011). [arXiv:1012.3512 [hep-th]].

[15] A. Kapustin, B. Willett, I. Yaakov, "Nonperturbative Tests of Three-Dimensional Dualities," JHEP 1010 (2010) 013. [arXiv:1003.5694 [hep-th]].

[16] A. Kapustin, B. Willett, I. Yaakov, "Tests of Seiberg-like Duality in Three Dimensions," [arXiv:1012.4021 [hep-th]].

[17] C. Krattenthaler, V. P. Spiridonov and G. S. Vartanov, "Superconformal indices of three-dimensional theories related by mirror symmetry," JHEP 1106 (2011) 008 [arXiv:1103.4075 [hep-th]].

[18] D. Gaiotto, E. Witten, "S-Duality of Boundary Conditions In N=4 Super Yang-Mills Theory," [arXiv:0807.3720 [hep-th]].

[19] A. Gadde, W. Yan, "Reducing the 4d Index to the S3 Partition Function," [arXiv:1104.2592 [hep-th]]. 
[20] A. Gadde, E. Pomoni, L. Rastelli and S. S. Razamat, "S-duality and 2d Topological QFT," JHEP 1003 (2010) 032 [arXiv:0910.2225 [hep-th]].

[21] A. Gadde, L. Rastelli, S. S. Razamat and W. Yan, "The 4d Superconformal Index from q-deformed 2d Yang-Mills," arXiv:1104.3850 [hep-th].

[22] F. A. H. Dolan, V. P. Spiridonov, G. S. Vartanov, "From 4d superconformal indices to 3d partition functions," [arXiv:1104.1787 [hep-th]]. 